94. 5022

Langenbecks

$\underset{\substack{\text { Aessindet } 1860 \\ \text { Archiv für Chirurgie }}}{ }$

Kongreßorgan der Deutschen Gesellschaft für Chirurgie

Kongreßband 1993

Redigiert von W. Hartel

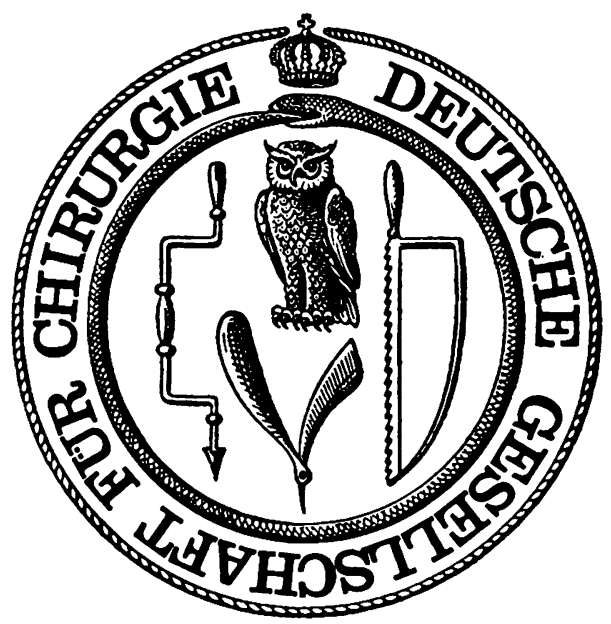




\section{Inhaltsverzeichnis/Contents}

\section{Eröffnungsansprache, Begrüßungsansprachen, Ehrungen, Mitgliederversammlung}

Eröffnungsansprache des Präsidenten

Präsident Prof. Dr. med. H. M. Becker, München 1

$\begin{array}{lr}\text { Begrüßungsansprachen } & 10\end{array}$

$\begin{array}{ll}\text { Totenehrung } & 18\end{array}$

$\begin{array}{lr}\text { Ehrungen } & 20\end{array}$

Preisverleihungen $\quad 25$

$\begin{array}{lr}\text { Mitgliederversammlung (Erster Teil) } & 29\end{array}$

$\begin{array}{ll}\text { Mitgliederversammlung (Zweiter Teil) } & 31\end{array}$

\section{Hauptthema}

Minimal invasive Chirurgie (MIC): Eine Bestandsaufnahme

1. Wandel der Chirurgie durch die MIC Minimal Invasive Surgery (G. Feifel)

2. Aus- und Weiterbildung in der minimal-invasiven Chirurgie (W. Hartel und H. P. Becker)

Education in Minimal Invasive Surgery

3. Ökonomische und strukturelle Folgen der MIC (H. Bauer)

Minimal Invasive Surgery (MIS) and its Effects on Economy and Structure of Health Care

4. Qualitätskontrolle im Rahmen der MIC (H. Hamelmann und O. A. Scheibe)

Surgical Quality Control MIS

5. Fehleranalyse - Evaluierung und Verhütung von Komplikationen; ihre juristische Implikation (H. Troidl, B. Bäcker, B. Langer und A. Winkler-Wilfurth)

6. Rechtliche Aspekte der MIC

(G. Carstensen)

7. Entwicklungsperspektiven der MIC Perspectives of Techniques of MIC (G. Buess)

Failure Analysis - Evaluation and

Prevention of Complications; its Juridical Implications 18

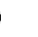
9 1 
9. Cholecystektomie - laparoskopisch versus konventionell
(A. Quentmeier und O. Scheibe)

10. Kosten und Kosten-Nutzen-Relation der MIC

(M. Rothmund)

11. Laparoskopische Eingriffe am Magen (K. Schönleben, I. Brune, G. Adamidis und F.-U. Zittel)

12. Laparoskopische Vagotomie (H.-F. Weiser und H. G. Klärner)

13. Laparoskopische Resektionen an Dünn- und Dickdarm

(E. Kraas, U. Kleine, A. Gemperle, E. Löhde und H. Loss)

14. Laparoskopische Eingriffe am Rektum (F. Köckerling, I. Gastinger, Th. Reck und B. Schneider)

15. Minimal-invasive Chirurgie in Kombination mit offener Chirurgie

(Ch. Klaiber, J. Tschudi, A. Metzger und $\mathrm{M}$. Wagner)

16. Minimal-invasive Oesophaguschirurgie (H. D. Becker)

17. Wandel im Therapiekonzept des Spontanpneumothorax - ein Vergleich konservativer mit konventionell operativer und thorakoskopischer Therapie

(M. Kästel, H. Wilkening, P. Bölcskei und Ch. Gebhardt)

18. Thorakoskopische Tumorchirurgie

(W. Wayand, R. Rieger und

$R$. Woisetschläger)

19. Laparoskopie beim akuten Abdomen (E. Eypasch, R. Mennigen, W. Spangenberger und $\mathrm{H}$. Troidl)

20. Appendektomie - Laparoskopisch vs konventionell

(E. Schippers)

21. Laparoskopische Verfahren beim Adhäsionsbauch

(G. Schaller, B. C. Manegold und M. Künkel)

22. MIC im Kindesalter (J. Waldschmidt)

23. Erfahrungen mit der laparoskopischen Vagotomie nach Taylor

(A. Keiler, Ch. Pernegger

und R. Hornof)
Laparoscopic Versus Conventional

Cholecystectomy

Costs and Cost-Benefit Relation of MIC

Laparoscopic Procedures in Gastric

Surgery

Laparoscopic Vagotomy

Laparoscopic Assisted Bowel

Resection

Laparoscopic Rectal Surgery

Minimal Invasive Surgery in Combination with Open Surgery

Changing in the Treatment of Spontaneous Pneumothorax - a Comparison of the Non-surgical Treatment With the Traditional Thoracotomie and with Minimal Invasive Therapy

Thoracoscopic Tumor Surgery

Laparoscopy for Acute Abdominal Emergencies

Appendicectomy - Laparoscopic vs Open

Laparoscopic Procedures

with Abdominal Adhesions

MIC in Infancy and Childhood

Experiences with Laparoscopic

Vagotomy by Taylor 
24. Die laparoskopische, extramuköse Pyloromyotomie: Ein kombiniertes laparoskopisch-endoskopisches Verfahren

(J. Schleef, M. Maragakis, K. Schaarschmidt und G. H. Willital)

25. Laparoskopische Behandlung einer Magenperforation mit einem Ethisorbstopfen

(H. Niebuhr, U. Nahrstedt, J. Holste und K. Rückert)

26. Minimal-invasive Chirurgie bei symptomatischen Milzzysten (T. Reck, F. Köckerling, I. Gastinger, B. Schneider und F. P. Gall)

27. Laparoskopische Therapie dysontogenetischer Lebercysten

(A. Emmermann, C. Zornig und M. Peiper)

28. Laparoskopische Splenektomie

(C. Zornig, A. Emmermann, M. Peiper und C. E. Broelsch)

29. Minimal-invasive Simultanoperationen (G. Meyer, V. Lange und F. W. Schildberg)

30. Ergebnisse der laparoskopischen Appendektomie im Vergleich zur konventionellen Operationstechnik (R. Raakow, H. Keck und P. Neuhaus)

31. Die transanale endoskopische mikrochirurgische Behandlung von ,low risk" und ,high risk" Karzinomen der Rektumschleimhaut

(A. Heintz, H. Menke, Th. Böttger und Th. Junginger)

32. Laparoskopisch assistierte Ileosacralresektion/Hemicolektomie rechts erste Erfahrungen

(F. Haaf, K.-H. Vestweber, M. Horatz und T. Weyer)

33. Minimal-invasive Chirurgie: Endoskopisch transthorakale Spondylodese bei traumatischer Bandscheibenläsion - Eine tierexperimentelle Studie (J. W. Maurer, J. Henke, St. Scharvogel und $\mathrm{H}$. Feussner)

34. Zeitliches Splitting im laparoskopischen OP-Plan bei Choledochuskonkrementen (J. M. Funovics, M. A. Funovics, Ph. Spießberger und G. Hochwarter)
The Laparoscopic Extramucous

Pyloromyotomia: a Combined Lapa-

roscopic Endoscopic Procedure

Laparoscopic Treatment of Stomach

Perforation by a Ethisorb Clog

\section{6}

Minimal Invasive Surgery in

Symptomatic Cysts of the Spleen

169

Laparoscopic Treatment of Dysontogenic Cysts of the Liver

Laparoscopic Splenectomy

Minimally-Invasive Simultaneous

Operations

Results of Laparoscopic Appendectomy in Comparison to Conventional Surgical Technique

The Transanal Endoscopic Microsurgical Treatment of "Low Risk" and "High Risk" Carcinomas of the Rectal Mucous Membrane

Minimal Invasive Surgery:

Transthoracic Endoscopic

Spondylodesis in Traumatic Lesions of the Intervertebral Disc

Therapeutical Splitting in

Laparoscopic Surgery of Common

Bile Duct Stones 
35. Was ist weniger invasiv bei der laparoskopischen Cholezystektomie: Die Hakenelektrode oder der Laser?

(R. Hoffmann, K. B. Brülhart,

R. Flury und F. Largiadèr)

36. Diagnostik und Therapie bei Gallengangsläsionen nach laparoskopischer Cholezystektomie

(Th. Manger und H. Wolff)

37. Minimal-invasive Thoraxchirurgie: Erweitertes Indikationsspektrum und verbesserte diagnostische Treffsicherheit der Thorakoskopie

(R. Bumm, H. Vogelsang

und H. W. Präuer)

38. Thorakoskopische Resektion von T1-Karzinomen der Lunge

(M. Hürtgen, K. Henneking, W. Padberg und K. Schwemmle)

39. Lungenrundherde unklarer Dignität und Stadien: verbesserte Diagnostik und Therapie mittels thorakoskopischer Resektion (R. Schwarz, C. Posner, P. F. Ferson und R. J. Landreneau)

\section{Kursus: Endosonographie}

40. Transösophageale Endosonographie (TEE) bei Herz- und Aortenerkrankungen

(J. L. Fischer und L. Henselmann)

41. Endosonographie bei Oesophagusund Magenerkrankungen

(H.-J. Dittler)

42. Endosonographie zur Beurteilung von Pankreas- und Gallenwegerkrankungen (T. Rösch)

43. Laparoskopische Sonographie Technik und Indikationen

(A. Brüggemann, T. Neufang und G. Lepsien)

44. Endosonographie des Kolons (G. Federmann)

45. Endosonographie bei Rektumtumoren (U. Hildebrandt)

46. Endosonographie bei entzündlichen anorektalen Prozessen

(B. Eibl-Eibesfeldt, S. Herzog

und S. Siebeck)
What is Less Invasive in Laparoscopic Cholecystectomy: Laser or Hook Electrode?

Diagnosis and Treatment of Lesions of the Common Bile Duct After Laparoscopic Cholecystectomy

Minimal Invasive Thoracic Surgery: Extended Indication and Increased

Diagnostic Security in Thoracoscopy

Thoracoscopic Resection

for T1 Lung Cancer

Circular Shadows of the Lung of Uncertain Dignity and Improved Staging Diagnosis and Treatment by Thoracoscopic Resection

Transesophageal Endosonography (TEE) in Heart- and Aortic Diseases

Role of Endoscopic Ultrasonography in Diseases of Esophagus and Stomach

Laparoscopic Ultrasound -

Equipment and Indications

Endosonography of the Colon

Endosonographic Findings in Pelvic and Anorectal Sepsis 
47. Endosonographie im Kindesalter Transrectal Ultrasound in Childhood (P. Dohrmann, M. Löhnert und W. Mengel)

\section{Weiter- und Fortbildung: Intraluminale endoskopische Chirurgie}

48. Intraluminäre endoskopische Chirurgie Intraluminar Endoscopic Surgery (B. C. Manegold)

49. Stents im Trachebronchialbereich (K. Häussinger)

50. Selbstexpandierende Stents in Ösophagus und Magen - eine Standortbestimmung

(G. Porse, U. Gerlach, D. Tübergen und B. C. Manegold)

51. Neues endoskopisches Behandlungsverfahren zur Palliation beim stenosierenden Rektumkarzinom (M. Dohmoto)

Self-Expanding Stents in Esophagus and Stomach - Current State

New Endoscopic Palliative Therapy with Prosthesis of Stenotic Rectal Carcinom

Acute Pancreatitis: Indication for ERCP and Papillotomy (M. Heinerman)

53. Die Sklerotherapie bei Ösophagusund Magenvarizen: heutiger Stand (K.-J. Paquet)

Sclerotherapy of Esophagogastric Varices: State of the Art

54. Sind chirurgische portosystemische Shunts noch indiziert?

(G. Otto, G. M. Richter, L. Theilmann und Ch. Herfarth)

Is Portosystemic Shunt Surgery Still Indicated?

55. Endoskopisches Vorgehen bei Fisteln im Bronchial- und Gastrointestinaltrakt (V. Lange, G. Meyer, G. Maiwald und T. Souvatzi)

56. Monitoring in der Endoskopie: was ist notwendig - was ist zuviel? (K.-D. Rückauer und R. Salm)

Endoscopic Intervention for Fistulae of the Bronchial-Tree and the Gastrointestinal-Tract

Monitoring in Endoscopic Examinations: What is Mandatory - What is Unnecessary?

\section{Hauptthema}

\section{Neues in der Chirurgie der Hernien}

57. Die Leistenhernie im Kindesalter (W. Lambrecht)

58. Verfahrenswahl: Bassini- versus Shouldice-Plastik in der Behandlung der Leistenhernie (U. Herzog, N. Demartines und $\mathrm{P}$. Tondelli)

59. Ergebnisse der Hernienchirurgie beim Erwachsenen

Inguinal Hernia in Infants and Children

Long-term Results After Transversalis Repair. A Comparison with Bassinirepair

Results of Inguinal Hernia Repair in Adults 
60. Laparoskopischer Hernienverschluß

(G. Lepsien, T. Neufang, M. Barthel und H.-J. Peiper)

61. McVay und Nyhus: Alternativen? (G. Ott, W. Dudda und E. Wenzel)

62. Ambulante Hernienchirurgie bei Erwachsenen - medizinische und sozialwirtschaftliche Aspekte

(J. Reydelet)

63. Verfahrenswahl bei Schenkelhernien (U. Fleck und W. Nowak $\dagger$ )

64. Das Hernienrezidiv

(M. Schmolke)

65. Das Faszienskelett der Bauchhöhle und die Hernien

(F. Stelzner)

66. Inzidenz, Pathogenese und Prophylaxe von Narbenhernien der Bauchwand

(R. Engemann, B. Lünstedt und

A. Thiede)

67. Indikation und Verfahrenswahl bei Narbenhernien

(V. Zumtobel und K. H. Bauer)

68. Die Inlay/Onlay-Technik als funktionelle Rekonstruktion der Bauchwand nach Narbenhernien und Narbenhernienrezidiven

(P. Klein, L. Wolff und R. Anetsberger)

69. Der problematische Bauchdeckenverschluß nach offener Lavagebehandlung (W. Teichmann und B. Herbig)

70. Neue Wege mit mikrochirurgisch transferierten innervierten Muskellappen zur Rekonstruktion der Bauchwandbrüche (A. Berger und W. Schneider)

71. Altes und Neues in der Behandlung angeborener Bauchwanddefekte im Kindesalter

(A. M. Holschneider, R. Richard und M. Gharib)

72. Die Shouldice-Reparation als Qualitätsmaßstab für Neues in der Hernienchirurgie: Fünf-Jahres-Ergebnisse der prospektiven Shouldice-Studie für Primär- und Rezidivhernien

(Ch. Töns, D. Kupczyk-Joeries,

F. P. Pfingsten und V. Schumpelick)
Laparoscopic Hernia Repair

McVay and Nyhus: Alternatives?

Ambulatory Surgical Treatment of

Hernia of Adults - Medical,

Economical and Social Point of View

Surgical Approach of Femoral

Hernias

Recidivation of Inguinal Hernia

The Fascial Skeleton of the Peritoneal

Cavity and the Hernias

Incidence, Pathogenesis and

Prophylaxis of Incitional Hernias

Indication and Operative Procedure in Incisional Hernias

Functional Reconstruction of the Abdominal Wall Following Hernia Using the Inlay/Onlay Technique

New Ways with Reinnervated Muscle Transfer by Microsurgical Methods for Reconstruction of the Abdominal Wall

New Aspects in the Treatment of Patients with Omphalocele and Gastroschisis

Shouldice-repair as Standard of Quality in Inguinal Hernia Surgery: Five Year Results in Primary and Recurrent Hernia 
73. Die Lokalanästhesie als Standardverfahren bei der LeistenhernienOperation (Ch. Peiper, Ch. Töns, F. Busch und V. Schumpelick)

74. Tageschirurgie - Hernienchirurgie beim Erwachsenen

(U. Muschaweck)

75. Leistenbruchoperationen nach Shouldice oder Bassini: Ergebnisse einer kontrollierten randomisierten Studie (A. Paul, H. Troidl, D. Rixen und J. Williams)

76. Laparoskopischer Nahtverschluß der Leistenbruchpforten (H.-J. Meyer)

77. Frühpostoperative Komplikationen nach Shouldice-Operation (R. E. Hilgert und A. Dörner)

78. Vermehrte Wundheilungsstörungen bei Leistenhernien durch Applikation der Thromboseprophylaxe in die Bauchdecken (H. Lemke, M. Imhoff und D. Löhlein)

79. Zusammenfassung der freien Vorträge: „Neues in der Chirurgie der Hernien I“" (J. Witte)

80. Laparoskopische Hernioplastik bei Rezidivhernien (T. Neufang, F.-E. Lüdtke und G. Lepsien)

81. Neues in der Chirurgie der Hernien Zusammenfassung der freien Vorträge (Sitzung Nr. 11)

(H. D. Saeger)
Local Anesthesia as Standard in Inguinal Hernia Repair

296

Outpatient Surgical Treatment

of Hernias in Adults

Inguinal Hernia Surgery After

Shouldice or Bassini: Results of a

Controlled Randomized Trial

Laparoscopic Surgery of Inguinal

Hernias

Early Complications on Inguinal Hernia Surgery: A Prospective Study of 200 Cases

Increased Healing Complications in Inguinal Hernia Surgery by Subcutaneous Abdominal Administration of Heparin as a Thromboembolic Prophylaxis

Summary of the Short Communications: "Currents State in the Surgery of Inguinal Hernia I"

Laparoscopic Hernioplasty in Recurrent Hernias: Special Technique, Casuistics and Preliminary Results

Surgical Hernia Repair - Summary of the Free Paper Session No. 11

\section{Hauptthema}

\section{Wandel in der Behandlung traumatischer Blutungen}

82. Die präklinische Versorgung innerer und äußerer Massenblutungen (J. Sturm)

83. Wandlung in der Behandlung traumatischer intracranieller Blutungen

(R. Fahlbusch, R. Laumer, U. Nissen, G. Schiffel und C. Cedzich)

84. Behandlung von Blutungen im Kieferund Gesichtsbereich (J.-E. Hausamen und R. Schmelzeisen)
Emergency Treatment of Massive Bleeding

Changes in the Management of Traumatic Intracranial Hemorrhage

Acute Control of Head and Neck Bleedings 
85. Vorgehen bei traumatischer Aortenrup-

tur

(L. Lauterjung)

86. Verletzungen von peripheren Arterien

und Venen

(S. Weimann)

87. Wandel in der Behandlung traumatischer Blutungen - Blutungen im Halsund Thoraxbereich

(K. P. Schmit-Neuerburg,

U. Obertacke, H. R. Zerkowski

und J. Chr. Reidemeister)

88. Wandel in der Behandlung traumatischer Blutungen: Leberrupturen

(B. Kremer)

Management of Traumatic

Hemorrhage from Injuries to Neck and Chest

89. Milzrupturen

(A. Encke und T. Weber)

Splenic Trauma

90. Blutungen bei Verletzungen

des Harntrakts

(H. Frohmüller und M. Theiß)

Surgery in Hepatic Trauma

1. Blutungen bei komplexen Beckenverletzungen

(H. Tscherne, U. Bosch und

T. Pohlemann)

92. Ausheilungsergebnisse nach konservativer Behandlung traumatischer kindlicher Milzrupturen

(A. Olinger, G. Schüder, A. Pizanis und R. Kubale)

93. Indikation zur konservativen Behandlung von Leberverletzungen

(J. Sturm, M. Raute und M. Trede)

94. Therapie der Leberverletzung agressiv oder konservativ

(M. Varney, H. Becker

und H.-D. Röher)

Results of Non-surgical Treatment of Traumatic Ruptures of the Spleen in Children

Bleeding in Patients with Urinary

Tract Injuries

Bleedings in Complex Pelvic Injuries

Indication for Conservative Treatment of Liver Injuries

Management of Liver Trauma:

Aggressive or Conservative

95. Behandlung der schweren Leberruptur durch Hepatectomie und Lebertransplantation

(B. Ringe, H. J. Meyer, R. Raab

und R. Pichlmayr)

96. Behandlung traumatischer Blutungen mittels interventioneller radiologischer Verfahren (L. Defreyne, P. Walter, R. Kubale und B. Kramann)

97. Risiken der Bluttransfusion

(V. Kretschmer)

Risks of Blood Transfusion 
98. Rechtliche Aspekte der Bluttransfusion (W. Weißauer)

99. Indikation zur Bluttransfusion und Strategien zur Vermeidung der Bluttransfusion

(L. Frey und K. Meßmer)

100. Die „Hypervolämische Hämodilution“ (HHD): Eine einfache Alternative zur isovolämischen Variante (E. Entholzner, S. Hargasser, L. Mielke und R. Hipp)

101. Maschinelle Autotransfusion im Baukastenprinzip

(P. Geiger, M. Gelowicz, H.-H. Mehrkens und K. H. Wollinsky)

102. Volumenersatz bei der präoperativen Eigenblutspende von Patienten mit kardiovaskulären Risikofaktoren (S.-M. Kasper, H. Dahlmann, W. Gerlich und D. Beiten)

103. Das „Concept Autologe Transfusion“" (CAT) - Kombination fremdblutsparender Maßnahmen zur Steigerung der Effektivität

(G. Singbartl und W. Schleinzer)

104. Bluttransfusions-assoziierte Immunsuppression als Risikofaktor beim kolorektalen Karzinom - Erste Ergebnisse einer randomisierten Studie (M. Heiss, Ch. Delanoff, K.-W. Jauch, W. Mempel und F. W. Schildberg)

105. Einsatzmöglichkeiten von Erythropoietin bei der Eigenblutspende (F. Mercuriali, G. Inghilleri, E., Biffi, A. Vinci, M. T. Colotti und R. Scalamogna)
Medico-legal Aspects of Blood

Transfusion

Indications for Blood Transfusion and Strategies to Avoid Transfusions

"Hypervolemic Hemodilution"

(HHD): A Simple Alternative to Normovolemic Hemodilution

Mechanical Autotransfusion in Modular Construction Design

Autologous Blood Donation: Volume Replacement After Phlebotomy in Patients with Cardiovascular Disease

"Concept of Autologous Transfusion" (CAT) - Increased Effectivity by

Combination of Different Autologous Transfusion Techniques

Erythropoietin and Autologous

Blood (AB) Transfusion to Minimize Homologous Blood (HB) Transfusion in Surgical Patients

Introduction to the Topic "Limits of Surgical Action"

Limits of Surgical Interventions and Therapy in Intensive Care Medicine Therapieabbruch in der Intensivtherapie (P. Lawin) 
108. Grenzen chirurgischen Handelns aus ethischer Sicht (J. Gründel)

109. Grenzen chirurgischen Handelns aus der Sicht des Patienten (G. Hoffmann)

110. Grenzen chirurgischen Handelns aus sozio-ökonomischer Sicht (H. Sitzmann)

111. Leben- und Sterbenlassen in der Chirurgie

(L. Schweiberer)

112. Grenzen chirurgischen Handelns in der Kinderchirurgie

(B. Thomasson)

113. Grenzen in der Notfallmedizin und Reanimation

(K. van Ackern)

114. Grenzen des herzchirurgischen Handelns

(B. Reichart, H. Netz, Ch. Schmitz, H. Mair, Ch. Detter, H. Dienemann und $\mathrm{H}$. Forst)

115. Grenzen chirurgischen Handelns in der Gefäßchirurgie

(H. Müller-Wiefel)

116. Grenzen chirurgischen Handelns beim Polytrauma

(G. Muhr)

117. Grenzen des chirurgischen Handelns bei multimodaler Tumortherapie (P. M. Schlag)

118. Grenzen chirurgischen Handelns in der Transplantationschirurgie

(C. E. Broelsch, X. Rogiers, M. Gundlach, W. Knoefel, R. Kuhlencordt, T. Langwieler und M. Greeve)

119. Grenzen der Brusterhaltung beim Mammakarzinom (F. Harder und U. Laffer)

120. Grenzen der Behandlung des Bronchialkarzinoms (H. Pichlmaier)

121. Grenzen chirurgischen Handelns beim Oesophaguscarcinom (J. R. Siewert)

122. Grenzen chirurgischen Handelns beim Lebercarcinom (P. Neuhaus und G. Blumhardt)
The Limits of Surgical Action From the Ethical Point of View

The Limitations of Surgery from the Patients Point of View

Limits on Surgical Treatment:

Social Economic Aspects

Live and Let Die in Surgery

408

On the Ethical Limits of Paediatric Surgery

Limitations within the Field of Cardiac Surgery

Limits of Operative Activities in Vascular Surgery

Limits of Surgical Action in Multimodality Cancer Treatment

Limits on Surgery in Transplantation

Limits of Conservative Treatment for Breast Cancer

Limits on Surgical Treatment for Bronchial Carcinoma

Limits to Surgery - in Esophageal Carcinoma

Limitations of Surgical Therapy in the Treatment of Primary Liver Cancer 
123. Grenzen chirurgischen Handelns bei Gallenwegstumoren

(K. D. Rumpf)

124. Grenzen chirurgischen Handelns beim Pankreaskarzinom

(M. Büchler, M. Ebert und H. G. Beger)

125. Grenzen der erweiterten und multivisceralen Resektion beim Magen- und Colonkarzinom (F. P. Gall)

126. Grenzen der Kontinenzerhaltung beim Rektumkarzinom

(R. Schiessel)

127. Grenzen chirurgischen Handelns in der Metastasenchirurgie (F. W. Schildberg, G. Meyer, G. Maiwald und H. G. Koebe)

128. Grenzen chirurgischen Handelns in der Tumortherapie - kritische Zusammenfassung (H. G. Beger)

129. Defektrekonstruktion an den cervicalen Visceralorganen nach Resektion organüberschreitender Schilddrüsencarcinome

(H. Dralle und G. F. W. Scheumann)

130. Wann ist die Resektion haematogener Melanommetastasen gerechtfertigt? (U. Krause, J. Friedrich und S. Assenmacher)

131. Multimodale Therapie des metastasierenden malignen Melanoms: Resektion residualen Tumors nach Immuntherapie mit Interleukin-2 und Interferon- $\alpha$

(E. Stoelben, H.-D. Saeger,

C. Scheibenbogen, W. Tilgen, J. Schmoll und U. Keilholz)

132. Grenzen chirurgischer Behandlungsmöglichkeiten von Desmoidtumoren bei familiärer adenomatöser Polyposis (FAP)

(G. Möslein, H. Buhr, M. Kadmon und $\mathrm{Ch}$. Herfarth)

133. Desobliterations-Operationen bei Tumorobstruktionen der Vena cava inferior. Die Grenze chirurgischen Handelns?

(G. F. W. Scheumann, C. Schmid, E. Allhoff und J. Laas)
Limitations in Surgery of Gall-DuctTumors

Limits of Surgical Therapy

in Pancreatic Cancer

Limits of Extended and Multivisceral

Resection in Gastric and Colonic

Cancer

Limitations of Sphincter Preservation

in Rectal Cancer

Limits in the Surgical Treatment

of Metastases

Critical Summary of the Session

About Limitations of Surgical Treatment for Malignant Tumors

Cervicovisceral Reconstruction After

Resection of Locally Thyroid

Carcinoma

When is Surgical Resection Justified in Hematogenous Metastases of Malignant Melanoma

Surgery of Metastatic Melanoma Following Successful IL-2 Based

Immunotherapy

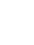


134. Die Kontinenzerhaltung durch koloanale Anastomose beim tiefen Rektumkarzinom - Ein Grenzbereich der Rektumchirurgie?

(K. Peitgen, M. K. Walz, J. Friedrich und F. W. Eigler)

135. Grenzen der Chirurgie bei der Resektion des Pankreaskarzinoms (H. Lippert)

136. Grenzen chirurgischen Handelns: Pfortaderresektion beim Pankreaskopftumor (J. D. Roder, J. Adolf, K. Böttcher und J. R. Siewert)

137. Grenzen der Lebertransplantation in der Therapie von malignen Tumoren (J. C. Rückert, Th. Manger, K. Gellert, G. Staffa, H. Lippert und H. Wolff)

138. Grenzen der Lebertransplantation bei Malignomen der Leber X. Rogiers, W. T. Knoefel, R. Kuhlencordt, M. Gundlach, T. E. Langwieler und C. E. Broelsch)

139. Probleme ökonomischer Bewertung medizinischer Therapieverfahren am Beispiel der Transplantationschirurgie (E. Nagel, K. D. Henke,

J. M. Graf v.d. Schulenburg,

F. W. Schwartz und R. Pichlmayr)

140. Nierentransplantation im höheren Lebensalter: Gibt es eine Altersgrenze? (P. Petersen, B. Hauerwaas, W.-D. Illner, G. O. Hofmann, K. Burkhardt, G. Hillebrand und W. Land)

141. Risikoabschätzung für die Nierentransplantation hochsensibilisierter Empfänger

(B. Greger, M. Kodsi und W. Lauchart)

142. Pankreas - nach vorausgegangener Nierentransplantation - eine Alternative zur simultanen Pankreas-/Nierentransplantation

(R. Grüßner, C. Troppmann,

A. Grüßner und D. Sutherland)

143. Grenzen der Indikation zur Intensivtherapie bei chirurgischen Patienten Prospektive Untersuchung zur Bedeutung von Scores (APACHE II, HIS, TISS) und anderer Faktoren für die Indikationsstellung zur Intensivtherapie (W. Wahl, K. Pelletier und T. Junginger)
Preservation of Recto-Anal

Continence by Colo-Anal

Anastomosis in Deep Rectal Cancer:

Limits on Rectal Surgery?

Limits on Surgery for Pancreatic

Cancer

Is Resection of the Portal Vein the Limiting Factor in Surgery of Carcinoma of the Head of the Pancreas?

Limits on Liver Transplantation in Treatment of Malignant Tumors

Limits on Liver Transplantation in Malignant Liver Tumors

Problems of Economic Assessment of Medical Treatment Procedures in Respect to Transplantation Surgery

Renal Transplantation in Advanced Age: Is there a Limit?

Risk Assessment of Kidney Transplantation in Highly Sensibilized Recipients

Pancreas Transplantation After Previous Kidney Transplantation: An Alternative to Simultaneous Pancreasand Kidney Transplantation?

Limits on Intensive Care in Surgical Patients: Prospective Study Concerning Significance of Scores and Further Factor for Affirmation or Refusal of Intensive Care Therapy 
144. Grenzen chirurgischen Handelns? Zum Krankheitsverlauf alter Patienten auf der Intensivstation

(J. Windolf, U. Eisele, R. Inglis und E. Hanisch)

145. Zur Operationsindikation bei AIDSKranken am Beispiel vollimplantierbarer intravenöser Portsysteme (Th. Rack, M. K. Walz, N. H. Brockmeyer, U. Krause und F.-W. Eigler)

146. Elektive und akute operative Eingriffe bei HIV-serokonvertierten Patienten; OP-Indikationen und postoperative Verläufe

(U. J. Hesse, W. Keil, M. Schrappe,

G. Fätkenheuer, B. Salzberger und R. Huber)

147. Ausgedehnte Darmresektionen im Rahmen des akuten Mesenterialgefäßverschlusses

(W. Meyer, J. Kolb und Ch. Gebhardt)

148. Grenzen der Effektivität chirurgischer Therapie des adrenalen Hypertonus (D. Simon, A. Lollert, P. E. Goretzki und H. D. Röher)
Limitations of Surgical Treatment? -

On the Clinical Course of Elderly

Patients in the ICU

Indication for Surgery in AIDS-

Patients: Implantable i.v. Port

Systems

Elective and Emergency Surgery

in HIV Saroconverted Patients:

Indications for Surgery and

Postoperative Outcome

544

Massive Bowel Resection in Patients

with Acute Mesenteric Ischemia

546

Limits on Effectiveness of Surgical

Treatment of Adrenal Hypertension

\section{Hauptthema}

Die kritische Extremitätenischämie - eine interdisziplinäre Herausforderung

149. Definition, Risikofaktoren und klinisches Bild der kritischen

Extremitätenischämie

(H.-J. Florek)

150. Die Pathophysiologie der kritischen

Ischämie

(G. Rudofsky)

151. Aktuelle spezielle Diagnostik der kritischen Beinischämie

(A. Scheffler und H. Rieger)

152. Aktuelle spezielle Diagnostik der kritischen Beinischämie (A. Scheffler, A. E., H. Rieger)

153. Chirurgische Möglichkeiten beim Mehretagenverschluß und isolierter infragenualer Okklusion (W. Sandmann)

Definition, Risk Factors and Clinical Symptoms of the Critical Leg Ischaemia

Pathophysiology of Critical Limb Ischaemia

Current Approaches to Laboratory Diagnosis of Critical Limb Ischaemia

154. Indikation zur Amputation bei kritischer Beinischämie (A. Huber und D. Rühland)
Indication for Amputation Due to Critical Ischemia of the Leg 
155. Die Amputation als primäre oder sekundäre Behandlung der kritischen Beinischämie (R. Baumgartner)

156. Was leistet die Pharmakotherapie allein oder additiv zur Chirurgie?

(H. Heidrich)

157. Epidurale Rückenmarksstimulation beim austherapierten arteriellen Verschlußleiden

(S. Horsch, L. Claeys und K. Ktenidis)

158. Periphere Nervus-ischiadicus-Blockade: Indikation und Ergebnisse

(B. Arnold, J. Hatzl, U. Weis-Walter und U. Jensen)

159. Peripherer Gefäßverschluß - Bypass oder Amputation?

(H. Erasmi, M. Walter, R. Schmidt und A. Prokop)

160. Thrombo-Embolektomie und Lyse in der Behandlung der kritischen Extremitätenischämie

(B. Steckmeier, G. Küffer und F. A. Spengel)

161. Was leistet die Profundarevaskularisation beim Zwei-Etagen-Verschluß im Stadium III/IV?

(R. Horstmann, A. Holzgreve, M. Kern und G. Hohlbach)

162. Der Arteria-tibialis-anterior-Bypass (G. Nagel, A. Scheidt und C. Käufer)

163. Intraoperative Bestimmung des Abflußwiderstandes als Parameter zur Prognosebeurteilung in der kruralen und pedalen Arterienchirurgie (W. Hepp, J. Frank, V. Henneken, U. Scholz und E. Hennig)

164. Die quantitative Analyse der kritischen Extremitätenischämie (D. Pennig)

165. Verbesserung der postischämischen Muskelfunktion durch geringe Reduktion der intramuskulären Temperatur während Ischämie (P.-M. Sutter, A. Marx, S. Dierauer, S. Strebel, J. Landmann und $\mathrm{M}$. Heberer)
Amputation as a Primary or

Secondary Procedure in Critical

Lower Limb Ischemia

What can we Expect from

Pharmacotherapy Applied Alone

or as an Adjunct to Surgery?

Epidural Spinal Cord Stimulation in the Treatment of Severe Peripheral Arterial Occlusive Disease

Peripheral Sciatic Nerve Block:

Indication and Results

Peripherial Vascular Disease - Bypass or Amputation?

Thromboembolectomy and

Thrombolysis in Treatment of Critical Limb Ischaemia

What do we Achieve by Revascularisation of the Profunda Femoral Artery in Multilevel Disease when Limb Threatening Ischemia is Present?

The Extraanatomic Anterior Tibial Bypass

Intraoperative Assessment of Run-off Resistance for Evaluation of Prognosis in Crural and Pedal Arterial Surgery

Quantitative Analysis of Critical Limb Ischaemia

Improvement of Postischemic Muscle Function by Slight Reduction of Intramuscular Temperature During Ischemia 
166. Die Beeinflussung der Lebensqualität durch Behandlung der kritischen Extremitätenischämie

(K. Brachmann und D. Falkenhahn)
Effect on Life Quality of Treatment of Critical Limb Ischaemia

\section{Kinderchirurgie}

\section{Analatresien}

167. Analatresie - Von der Grundlagenforschung zur funktionsgerechten Operation (D. Kluth, P. Reich und W. Lambrecht)

168. Analatresien - eine klinische Übersicht (W. Schubert a.E. und D. Roesner)

169. Assoziierte Syndrome bei anorektalen Fehlbildungen: Restriktivere Stellung der Operationsindikation? (H. P. Hümmer, S. Simon und P. Klein)

Ano-rectal Malformations Impact of Basic Research on Surgical Procedure

Anal Atresie - A Clinical Review

Restrictive Indication for Surgery in Syndromatic Anorectal Malformations?

170. Vergleich des Operationserfolges nach Korrektur einer anorektalen Mißbildung in Abhängigkeit des durchgeführten Eingriffs (L. M. Wessel, S. Hosie, K.-L. Waag und A. Ballauff)

171. Myokinetischer Sphinkterersatz durch Glutaeus maximus bei hoher Analatresie (J. Ch. Braun, J. Starke $\dagger$, G. Steinau und V. Schumpelick)

Evaluation of the Surgical Success of Different Operative Strategies in the Correlation of Anorectal Malformations

Reconstruction of Rectal Sphincter by Transposition of Gluteus Muscle

172. Korrektur einer hohen Kloakenfehlbildung (A. M. Holschneider und H. Hendren)

Repair of a Cloacal Malformation

\section{Zwerchfellhernien und Defekte}

173. Zwerchfellhernien Eine klinische Übersicht (K. Gdanietz)

Diaphragmatic Hernias A Clinical Survey

174. Rechtsseitige Zwerchfellbrüche Wert der Sonographie (R. Daum und H. Roth)

Right Sided Diaphragma Hernias Importance of the Ultrasound

175. Erfahrungen in der Diagnostik und Therapie von $Z$ werchfell-Lücken und Zwerchfellhernien im Säuglingsalter (W. Tischer, J. Bennek, K. Rothe und D. Brock)

Our Experience in Diagnosis and Treatment of Diaphragmatic Defects and Diaphragmatic Hernias in Infants 
176. Strategiewandel in der Behandlung von kongenitalen Zwerchfellhernien (W. Löffler, H. Roth, R. Daum, E. Zilow und H. Leonhardt)

177. Die Embryologie des Zwerchfellverschlusses (Video-Film)

(D. Kluth, W. Lambrecht, P. Reich und F. Schier)

178. Die Pathogenese der Lungenhypoplasie bei angeborenen postero-lateralen Zwerchfelldefekten

(M. v. Ekesparre, D. Kluth, P. Reich und W. Lambrecht)

179. Die Lungenhypoplasie und ihre Auswirkung auf die Prognose der konnatalen Zwerchfellhernie (Ch. Deindl und E. Pensel-Göttle)

180. Extracorporale Membranoxygenierung (ECMO) bei der Behandlung von angeborenen Zwerchfellhernien (H. Wirth, W. Brands, W. Kachel und K.-L. Waag)

181. Tierexperimentelle Untersuchungen an Ratten mit verschiedenen Materialien zur Zwerchfellkonstruktion (G. Steinau, A. Schindler, J. Braun und V. Schumpelick)

\section{Plastische Chirurgie}

\section{Langzeitergebnisse nach Replantation}

182. Beurteilungskriterien für und nach Replantationen

(P. Brenner und A. Berger)

183. Langzeitergebnisse nach Daumenreplantation

(B. Landsleitner)

184. Langzeitergebnisse nach Einzelund Mehrfingerreplantationen (E. Biemer, P. Graf, R. Gröner und K. Biefel)

185. Heterotope Replantation und ihre Langzeitergebnisse

(B.-D. Partecke)

186. Spätergebnisse nach Replantation im Kindesalter

(G. Meissl, H. Piza und L. Walzer)
Change of Strategy in Treatment of Congenital Diaphragmatic Hernia

Embryology of the Diaphragm,

Closure of the Dorsolateral Region

The Pathogenesis of Pulmonary Hypoplasia $(\mathrm{PH})$ Associated with Congenital Diaphragmatic Hernia $(\mathrm{CDH})$

Extracorporeal Membrane

Oxygenation in the Treatment of

Congenital Diaphragmatic Hernias

Prosthetic Materials and Muscle Flap in the Repair of Diaphragmatic Defects: An Experimental Study

Criterion for and Functional Assessment Following Replantations

Longterm Results After ThumbReplantation

Long-Term Results After Single or Multiple Finger Replantation

Long-Term-Results of Heterotopic Replantations

Long-Term Results After Replantation in Children 
187. Langzeitergebnisse nach Replantation von Extremitätenteilen

(R. G. H. Baumeister, T. Hofmann und A. Frick)

188. Langzeitergebnisse nach Replantation von Extremitäten

(P. C. Maurer, S. Strobl, G. Pflugbeil,

St. von Sommoggy, P. Heider und

P. R. Graf)
Long-Term Follow-up After

Replantation of Parts of Extremities

705

Long-Term Results After Limb-

Replantations

Rekonstruktive Eingriffe im Gesichtsbereich nach Tumor und Trauma

189. Rekonstruktive Eingriffe im Gesichtsbereich nach Tumor und Trauma.

Grundprinzipien der Wiederherstellung

(G. M. Lösch)

190. Rekonstruktion der Haut und Weichteile nach Tumoren im Gesichtsbereich (M. Greulich und W. Gubisch)

191. Rekonstruktion von Haut- und Weichteilen nach Traumen

(G. Spilker und G. B. Stark)

192. Kombinierte Verfahren zur Wiederherstellung auch des knöchernen Skeletts (W. Stock und E. Dielert)

193. Vollhauttransplantate für Halsund Gesichtsrekonstruktion (R. Hettich, D. Kistler und S. Eren)

194. Rekonstruktive Eingriffe zur Wiederherstellung der Funktion und Mimik (W. Schneider und A. Berger)

195. Tumorrezidivchirurgie und extreme Tumorchirurgie im Gesichtsbereich welche Konsequenzen hat der Patient zu tragen?

(R. B. Drommer)

196. Der Stellenwert des myocutanen Pectoralis-major-Lappens in der rekonstruktiven Chirurgie im KieferGesichts-Bereich

(A. Bremerich, E. Machtens, W. Kozuschek und N.-C. Gellrich)

197. Zur Rekonstruktion des Unterkiefers: Entwicklung von Techniken in mehreren Jahrzehnten

(H.-D. Pape, K. L. Gerlach, K. E. Rehm und Ch. Schippers)
Basic Principles of Reconstruction

Reconstruction of the Skin and Soft

Tissue After Resection of Tumors

Reconstruction of Skin and Soft

Tissue After Trauma

Combined Methods of Reconstruction of the Bone Structures

Full Thickness Skin Grafts for Neck and Face Burn Reconstruction

Comprehensive Treatment of Patients with Extensive Malignant Tumors and Recurrent Tumors - What Can They Expect?

The Rank of Myocutaneous Pectoralis-Major Flap in Reconstructive Surgery of Jaw and Face

Concerning Reconstruction of the Mandibula: Development of Techniques in Several Decades 
198. Dreidimensionale Rekonstruktion von schweren Gesichtsschädelfrakturen unter Zuhilfenahme moderner bildgebender Verfahren

(P. Stoll, R. Wächter, A. Nilles,

B. Wimmer und R. Kirchner)

199. Der koronale Zugang zur Rekonstruktion komplexer kranio-fazialer Traumen

(N. Hardt, A. Gottsauner und F. Sgier)
Three-Dimensional Reconstruction of Severe Fractures of the Facial Skull with the Aid of Modern Imaging Methods

The Coronal Access for Reconstruction of Severe Cranio-maxillo-facial Injuries

\section{Herzchirurgie}

Extrakorporale und assistierte Zirkulation

200. Die Physiologie der extrakorporalen und der assistierten Zirkulation (G. Hellige)

201. Extrakorporaler veno-venöser Langzeitbypass beim ARDS

(H. Lennartz)

202. Extrakorporale Kreislaufunterstützung bei prä- und postoperativem Herzversagen (R. Körfer, H. Posival und A. El-Banayosy)

Pre- and Postoperative Myocardial Failure and Mechanical Circulatory Support

203. Extrakorporale Kreislaufunterstützung und mechanische Überbrückung zur Herztransplantation (R. Hetzer, A. Schiessler, N. Friedel, Y. Weng und E. Hennig)

Extracorporeal Circulatory Assistance and Mechanical Bridging to Heart Transplantation

204. Intraaortale Ballongegenpulsation Indikationen, Risiken und Ergebnisse (P. Kalmár, H.-M. Stubbe und Chr. Rülke)

205. Die Anwendung der Zentrifugalpumpe in der Thorax-, Herz- und Gefäßchirurgie (F.-W. Mohr, R. Autschbach, J. Gummert und A. Diegeler)

Intra-aortic Balloon Counterpulsation

Use of Centrifugal Pumps in Cardiovascular Surgery

206. Extrakorporale Zirkulation in der Chirurgie der thorakalen Aortenaneurysmen

(M. Horst, A. Borowski, M. Südkamp und E. R. de Vivie)

207. Extrakorporale und assistierte Zirkulation bei der Lungentransplantation (A. Haverich, St. Hirt und J. Cremer)

Extracorporal Circulation in Surgery of the Thoracic Aortic Aneurysms

Extracorporeal and Assisted Circulation in Lung Transplantation

Extracorporal Perfusion in Liver Transplantation
208. Extracorporale Organperfusion der Leber

(H. J. C. Wenisch) 
209. Extrakorporale, kontrollierte Extremitäten-Reperfusion

(F. Beyersdorf, K. Sarai, Z. Mitrev,

K. Ihnken, G. Matheis, L. Eckel

und P. Satter)
Extracorporeal, Controlled Limb

Reperfusion

Extrakorporale und assistierte Zirkulation und thorakoskopische Chirurgie

210. Präoperative Risikofaktoren bei mecha- Preoperative Risk Factors in nischer Kreislaufassistenz als Überbrückung zur Herztransplantation (A. Schiessler, N. Friedel, E. Hennig und R. Hetzer)

Mechanical Circulation Assistance for Bridging Time in Patients Planned for Heart Transplantation

211. Die Anwendung des linksventrikulären Kreislaufunterstützungssystems Novacor als Überbrückung zur Herztransplantation

(H. O. Vetter, H. G. Kaulbach,

O. Gödje, H. Reichenspurner,

E. Kreuzer, G. Schindler, W. Röll

und B. Reichart)

212. Assistierte Zirkulation bei einem 7 Tage Assisted Circulation in a 7 Days Old alten Neugeborenen wegen Postkardiotomie-low-output-syndrom

(F. X. Schmid, U. Hake, B. Eberle,

D. Schranz und H. Oelert)

Application of the Left Ventricular Assist System NOVACAR as Bridging for Heart Transplantation

Newborn Infant for Postcardiotomy

Low Output Syndrome

\section{Thoraxchirurgie}

\section{Organerhaltende Operationen an der Lunge}

213. Organerhaltende Operationen an der Lunge - Aspekte der Pathologie (K.-M. Müller und A. Theile)

214. Die Bedeutung der interventionellen Bronchoskopie im Rahmen organerhaltender Operationen an der Lunge

(H. D. Becker)

215. Broncho- und angioplastische

Operationen beim Bronchial-Ca

(D. Branscheid, S. Trainer,

H. Bülzlebruck, S. Krysa

und I. Vogt-Moykopf)

216. Parenchymerhaltende BronchoplastEingriffe im Bereich des Bronchus intermedius

(P. Keszler)

217. Organerhaltende Eingriffe beim Bronchialkarzinom

(C. Engelmann)
Organ-serving Operations of the

Lung - Aspects of Pathology

Interventional Bronchoscopy in

Surgery of the Central Airways

Sparing Bronchoplastic Procedures at the Level of Bronchus Intermedius

Organ Preserving Operation in

Bronchial Carcinoma 
218. Organerhaltende Operationen bei benignen und semimalignen Erkrankungen

(H. Dienemann, H. Hoffmann, Ch. Müller und F. W. Schildberg)

219. Manschettenresektion bei Bronchusstenosen des Kindesalters: Indikationen, Vorgehen, Langzeitergebnisse (R. H. Richter, H. P. Hümmer, S. Simon, Th. Zimmermann, K.-R. Greskötter und F. P. Gall)

220. Spezielle Komplikationen nach organerhaltenden Eingriffen an der Lunge und deren Behandlung - Bronchoplastische Resektionen

(M. Semik, A. Linder, G. Horea, G. Friedel und H. Toomes)
Conservative Operations in Benign

Lesions and "Adenomas" of the Lung
Sleeve Resection for Bronchial Stenosis in Children: Indications, Procedure, Results of Long-term Follow-up

Special Complications and Feasible Therapy After Bronchoplastic Resections

\section{Unfallchirurgie}

Die Bedeutung der Biologie in der Traumatologie

221. Die Bedeutung der Biologie in der

Traumatologie -

Einleitende Bemerkungen zum Thema

(S. Weller)

222. Der Einfluß der Vitalfunktion auf die

Wahl des Osteosyntheseverfahrens

beim Schwerverletzten

(D. Trentz)

223. Der Einfluß des Weichteilschadens auf die Frakturheilung - ,Konzept der Funktionseinheit Weichteil-Knochen" (A. M. Betz, W. Stock, R. Hierner und L. Schweiberer)
The Importance of Biological Aspects in Traumatologie - Introductional Remarks to the Main Topic

Influence of Soft Tissue Defect on Fracture Healing - "Concept of the Functional Unit Soft-Tissue-Bone"

\section{Moderne Osteosynthesetechniken zur verbesserten Schonung} der Fragmentdurchblutung an Beispielen

224. Der Fixateur externe

(D. Höntzsch)

External Fixation

225. Die Bedeutung der Biologie

in der Traumatologie

(N. Haas, M. Schütz und U. Stöckle)

The Significance of Biology

in Trauma Treatment

226. Moderne Osteosyntheseverfahren zur verbesserten Schonung der Fragmentdurchblutung: Marknagelung

(C. Krettek, P. Schandelmaier, C. Pape und $\mathrm{H}$. Tscherne)

Unreamed Nailing Techniques

for Fixation of Long Bone Shaft

Fractures

227. Der biologische Knochenersatz (R. Brutscher)

Biological Bone Substitute 
228. Die Bedeutung der Biologie in der Traumatologie (A. Rüter)

229. Erkennen typischer opportunistischer Infektionen bei AIDS-Patienten vor unfallchirurgischen Akuteingriffen

(T. Kossmann, K. B. Brülhart, M. C. Morganti-Kossmann und $\mathrm{O}$. Trentz)

230. Versorgung kindlicher Schaftfrakturen mit dem Fixateur externe (J. Bennek, D. Brock, K. Rothe und U. Bühligen)

231. Die ventrale tanspedunkuläre Instrumentation - eine neue Stabilisation nach Wirbelkörperresektion bei Metastasen der LWS (H. Hertlein, T. Mittlmeier, M. Schürmann und G. Lob)

232. Kontinuierliche Kompartmentdruckmessung bei Oberschenkelschaftfrakturen

(K. P. Lehrbaß-Sökeland, V. Golombek und M. Hansis)

233. Gewebeschonende Operationstaktik bei der Tibiakopffraktur (R. Volkmann, F. Maurer, S. Weller und $M$. Jockheck)

234. Chirurgisches Management bei kniegelenksnahen Frakturen und Luxationen mit kritischer Extremitätenischämie (W. Lang, G. Rümenapf, H.-P. Koerfgen und H. Schweiger)

235. Kompartment-Syndrom des Unterschenkels

(M. Walter, M. Gawenda, H. Erasmi und R. Schmidt)

236. Drehfehler nach Unterschenkelmarknagelung und Fixateur Externe (P. Schandelmaier, C. Krettek, E. Scola und $\mathrm{H}$. Tscherne)

237. Die Antibiotika-imprägnierte Spongiosaplastik als biologische Trägersubstanz in der OsteomyelitisBehandlung (K. Neumann, W. Buchholz und G. Muhr)

238. Therapie von infizierten Pilon-TibialeFrakturen mit dem Ilizarov-Verfahren (M. Raschke, G. Oedekoven, Ch. Freisleben und B. F. Claudi)
Importance of Biological Factors in Traumatology

Identification of Opportunistic Infections in AIDS Patients Before Traumatic Emergency Operations

The Application of a Unilateral External Fixator in Children with Shaft Fractures

Ventral Transpeduncular Instrumentation: a New Stabilization After Vertebral Body Resection of the Lumbar Spine for Metastases

Continuous Compartment Pressure Assessment in Femur Fractures

\section{8}

Tissue Preserving Operative Tactics in Tibial Head Fractures

Surgical Management in Fractures Near the Knee Joint Combined with Critical Limb Ischaemia

Compartment Syndrome of the Lower Limb

Malrotation After Intramedullary Nailing and External Fixation of Tibial Fractures

Antibiotic Impregnated Spongiosaplasty as Biologic Carrier Susbtance in Treatment of Osteomyelitis

Treatment of Infected Fractures of the Pilon Tibiale by the Ilizarov Procedure 
239. Oberschenkelfraktur bei liegender Hüftendoprothese - Plattenosteosynthese versus Wagner-Revisionsprothese (A. Lies, J. Buchholz, A. Ekkernkamp und Ch. Josten)

240. Primäre Oberschenkelmarknagelung bei Polytrauma mit Lungenkontusion - Erhöhtes ARDS-Risiko? (H.-C. Pape, G. Regel, M. Auf'm'kolk und $\mathrm{H}$. Tscherne)

241. Spongiosaplastik oder biologische Abstützung bei komplexen Frakturen des Oberschenkels

(K. Wenda, J. Degreif, M. Runkel und G. Ritter)

242. Konzept und Ergebnisse der primär komprimierten Veriegelungsnagelung ohne Kortikalisaufbohrung an Femur und Tibia

(V. Bühren, M. Potulski und I. Marzi)

243. Die Plattenosteosynthese bei der Tibiaschaftfraktur - wann ist sie unter biologischen Gesichtspunkten heute noch indiziert?

(M. Jockheck, R. Volkmann und

S. Weller)

244. Der unaufgebohrte Marknagel beim offenen Unterschenkelbruch - eine sinnvolle Alternative zum Fixateur externe?

(W. Knopp, P. A. W. Ostermann, J. Buchholz und G. Muhr)

245. Biologische Osteosynthesen von Schaftfrakturen der unteren Extremität im Wachstumsalter

(H. G. Dietz, P. P. Schmittenbecher, P. Knorr und M. Stehr)

246. Ergebnisse nach Minimalosteosynthese der Pilon-Tibial-Fraktur

(D. Nast-Kolb, A. Betz, Ch. Rödel und L. Schweiberer)

247. Einfluß der Behandlungsmodalitäten auf die Früh- und Langzeitergebnisse bei Pilon-Tibial-Frakturen

(Chr. Kuntz, W. Friedl und P. Meeder)

248. Ringfixateur oder unilateraler Fixateur - beeinflußt die unterschiedliche Montageform die Knochenregeneration beim Segmenttransport?

(Ch. Josten, A. Ekkernkamp, A. Lies

und G. Muhr)

Thigh Fracture After Total Hip

Endoprosthesis: Metal Osteosynthetic

Operations vs. Wagner Revisions

Prosthesis

Primary Intramedullary Nailing of

Femur Fractures in Polytraumatized

Patients with Lung Contusion:

Increased Risk of ARDS?

Bone Grafting or Biologic Butress

in Complex Fractures of the Femur?

Concept and Clinical Results of Primary Compressed Interlocking Nailing of the Femur and the Tibia

Plating in Tibial Fractures: Still Indicated Nowadays Regarding Biological Aspects?

The Undrilled Marrow Nail in Open Tibia Fractures: Useful Alternative to External Fixation?

"Biological Osteosynthesis" of Shaft Fractures of the Lower Limb in Children

Results of Minimal Osteosynthetic Procedures in Fracture of the Pilon Tibiale

Influence of Treatment Modalities on Early and Late Results of Pilon Tibial Fractures

Ring Fixateur or Unilateral Fixateur: Influence of the Different Form of Installation on Bone Regeneration? 
249. Die Plattenosteosynthese proximaler Humerusfrakturen in der No-touch-

Technik

(C. Dahlen, J. Henkel und L. Gotzen)

250. Die Fixateur-externe-Osteosynthese

The External Fixation in Fractures of distaler Radiusfrakturen nach dem the Distal End of the Radius Using Prinzip der Ligamentotaxis (K. Huch, M. Hünerbein und P. J. Meeder)

\section{Gefäßchirurgie}

Maßnahmen zur Qualitätsverbesserung und -sicherung in der Gefäßchirurgie: Nachbehandlung und Nachkontrollen

251. Gefäßrekonstruktion und Rehabilitation (W. Schoop)

252. Postoperative Kontrollintervalle nach Gefäßrekonstruktionen: Welche Parameter sind zu überprüfen? (M. Haug, F. Frizen und G. Krüger)

253. Medikamentöse Nachbehandlung nach peripherer Gefäßrekonstruktion (H. Kortmann, M. Sokol und T. Mansfeld)

254. Postoperative sonographische Verlaufskontrollen nach aortalen und femoropoplitealen Rekonstruktionen
(A. Schröder, G. Riepe,
G. v. Klinggräff und H. Imig)

255. Kontrollangiographie - Indikation und Verfahrenswahl: Ist die DSA ausreichend?

(G. Hagmüller, M. Hold und A. Schlegl)

256. Computergestützte Nachsorgesysteme (O. Wagner und M. Schemper)

257. Service-Operationen oder interventionelles Vorgehen bei Rezidivstenosen nach peripherer Gefäßrekonstruktion (J. Largiader und E. Schneider)

\section{Carotischirurgie}

258. Zur Wertigkeit der intraoperativen transkraniellen Dopplersonographie (F. M. Grögler und H. J. v. Büdingen)

259. Wert der Stumpfdruckmessung in der Carotis-Chirurgie (S. Hutschenreiter)
Vascular Reconstruction and

Rehabilitation

Postreconstruction Follow-ups in

Vascular Surgery: Why? Who? When? What?

Medical Treatment After Distal

Arterial Reconstruction

Postoperative Sonography in Surveillance of Aortic and Femoropopliteal Reconstructive Surgery

Controllangiography: Indication and Choice of Method: Is i.v. DSA Sufficient?

Computer Aided Follow-up Systems 988

The Value of the Stump Pressure Measurement in Carotid Surgery 
260. Wert der somatosensorischen evozierten Potentiale (SEP) und des EEG-Monitorings als intraoperative Kontrolle bei Carotisoperationen (H. W. Kniemeyer, R. Kolvenbach und W. Sandmann)

261. Wert der Gefäßendoskopie in der Carotischirurgie (P. Kasprzak und D. Raithel)

Value of Somatosensory Evoked Potentials and EEG Monitoring in Intraoperative Control During Carotid Operations

Value of Angioscopy in Carotid Surgery

Are Peripheral Arterial Reconstructions Justified Without Intraoperative Control Angiography

Appraisal of Vascular Endoscopy for Monitoring Infrainguinal Bypass Grafts

1004

Prognostic Value of Peripheral Resistance Measurement for Graft Function

(H. Schweiger und W. Lang)

Hemodynamic Monitoring in Aortic Reconstructive Surgery (H. Böhrer und E. Martin)

PTA and ITA of the Aortic Branches and Iliac Arteries und Beckenarterien (J.-R. Allenberg und G. Richter)

267. PTA und ITA der unteren Extremitäten (D. Rühland)

PTA and ITA of Femoral Arteries PTA of Brachio-cephalic Arteries

268. PTA der supra-aortalen Arterien (R. Kachel)

269. Simpson-Atherektomie-Katheter

(B. Höfling)

270. Rotationskatheter (G. Küffer)

Rotational Devices

271. Laserangioplastie (H. Berger, C. Hundt, P. Kohz und L. Lauterjung) 
272. Aspirationsthrombembolektomie (F.-J. Roth und S.-Ph. Roth)

273. Intraarterielle Lyse

(G. Hohlbach)

274. Vaskuläre Stent-Implantationen

(W. Gross-Fengels, H. Imig,

W. Heindel und A. Schröder)

275. Kavafilterimplantation

(D. Vorwerk, R. W. Günther und J. Neuerburg)

276. Transjugulärer intrahepatischer portocavaler Shunt (TIPS)

(G. Noeldge)

277. Intravasale Sonographie

(J. M. Heiss, H. Rinecker

und R. Kreuzer)

278. Angioskopie

(H. Loeprecht)
Aspiration Thrombembolectomy

Intraarterial Lysis

Vascular Stent-Implantations

Implantation of Caval Filters

Intravascular Ultrasound

1022

1023

EDV in der Chirurgie: Anwendungsbeispiele Gefäßchirurgie

Data Bank in a Vascular Surgery

Department

(J. Sauer, P. Heider

und St. v. Sommoggy)

1023

280. EDV-Dokumentation in Grebenhain

(G. Stelzer)

1023

EDV-Documentation in Augsburg

1024

Patient Documentation in Vascular

Surgery with Windows-Superbase 4

base 4 in der Gefäßchirurgie

(A. Herrmann, K.-H. Orend,

B. Fetscher und L. Sunder-Plassmann)

283. Stand der EDV im Städtischen

Krankenhaus München-Neuperlach

(H. Niedermeier und F. Furtschegger)

284. Die perioperative Dokumentation

mit ANGIOBASE

(H.-J. Schober-Halstenberg

und H.-D. Clevert)

In Zusammenarbeit mit dem Berufsverband:

Anforderungsprofil mit der Position des leitenden Krankenhauschirurgen

285. Anforderungsprofil des leitenden Arztes

im Krankenhaus der Grund- und

Regelversorgung

(H. Hempel) 
286. Anforderungsprofil in der Position des leitenden Krankenhauschirurgen aus Sicht des Universitätschirurgen (J. R. Siewert)

287. Anforderungsprofil in der Position des leitenden Krankenhauschirurgen: Aus der Sicht des Krankenhauschirurgen (H. Bauer)

288. Anforderungsprofil in der Position des leitenden Krankenhauschirurgen aus der Sicht des Krankenhausträgers (W. G. Fack-Asmuth)

289. Anforderungsprofil in der Position des leitenden Krankenhauschirurgen aus der Sicht des Kostenträgers

(H. Sitzmann)

290. Anforderungsprofil in der Position des leitenden Krankenhauschirurgen aus der Sicht des Arztrechts

(M. Andreas)

291. Anforderungsprofil in der Position des leitenden Krankenhauschirurgen aus der Sicht wirtschaftlicher und sozialrechtlicher Fragen - Ermächtigungen (J. Bauch)

292. OPTIMA-Leistungsmanagement in der Klinik

(E. Schmid)
Required Qualifications for the

Position as Head of a Community

Hospital - the University Surgeon's

View

Chief or Surgery:

Profile of Requested Abilities

Qualification of a Leading Hospital Surgeon from the Hospital Owner's

Point of View

From the Health Insurance Point of View

1044

From the Medical Profession's Law

Point of View

1047

Prerequisite for a Position of

Surgeons in Leading Hospital Areas

in View of Economic and Legal

Interpretations - Authorization

OPTIMA-Efficiency Management in the Clinic

\section{Weiter- und Fortbildung}

Intensivmedizin bei posttraumatischem Organversagen

293. Pathophysiologie des Organversagens

nach Trauma

(M. Nerlich)

294. Kreislaufversagen

(B. Schwarz und H. Zuckermann)

295. Respiratorisches Versagen

Respiratory Failure

(H. Bartels und J. R. Siewert)

296. Intensivmedizin bei posttraumatischem

Leberversagen

Intensive Care for Posttraumatic

(W. O. Bechstein)

297. Nierenversagen

Liver Failure

Acute Renal Failure

(D. Inthorn) 
298. Einführung zum Thema: Warum eine Verknüpfung der beiden Begriffe? (W. Lorenz)

299. Beziehung von prä- und intraoperativen Risikofaktoren bei der postoperativen Wiederherstellungsqualität

(Th. Junginger, $\mathrm{H}$. Menke und A. Klein)

300. Neue pathophysiologische Prozesse beim perioperativen Risiko: Auswirkungen auf die Qualitätssicherung (E. Neugebauer)

301. Mortalitätsraten: Beispiele für das Problem der Kausalität in der Qualitätssicherung

(E. Bollschweiler und J. R. Siewert)

302. Einfluß der Risikoanalyse auf den

Kreisprozeß der Qualitätssicherung:

Die tägliche Praxis

(O. Scheibe und P. Allhoff)

303. Qualitätssicherung bei großen Eingriffen

(I. Gastinger und H. Lippert)

304. Qualitätssicherung in einer neuen Dimension: Standardisierung der Behandlungsstrategien untereinander bei verschiedenen Kliniken (H.-K. Selbmann)

Introduction: Why is a Connection of the two Terms Intended?

Relation of Pre- and Intraoperative

Risk Factors to Postoperative

Recovery

New Pathophysiological Processes and Perioperative Risk: Consequences For Quality Assurance

Rates of Mortality: Examples for the Problem in Causality of Quality

Assurance

The Circle Process of Quality

Protection and its Influence on Risk

Analyses: The Daily Experience

Quality Control in Major Abdominal Surgery

Quality Assurance in a New

Dimension: Standardisation of Treatments by Cooperation of Hospitals

EDV in der klinischen Chirurgie: Datenerfassung und Dokumentation

Termoverlapping Dataprocessing for Perioperativ Quality Control

Clinical Information System

306. EDV-Dokumentation

(H. G. Kroczek, R. Bähr

und D. Laqua)

307. OP-Planung und OP-Betriebsablaufsteuerung als Teil des Klinik-Kommunikationssystems KMMS

(B. Pollwein, L. Gierl und H. Krämling)

308. Leistungserfassung im Hinblick auf Abrechnung und Budgetierung sowie Fallpauschalen (G. Neubauer)

Hospital-Output, Budgeting, and Case-based Hospital Reimbursement 
309. Die elektronische Krankenakte

(D. Hoelzel, K. Adelhard und W. Tretter)

310. Die digitale Krankengeschichte im klinischen Alltag (M. Fischer, G. Segmüller und G. Sennwald)

311. Chirurgische Praxis-EDV (G.-D. von Koschitzky)
The Computer-based Patient Record 1067

Electronic Patient Charts in Clinical Workaday Routine

Computer in a Surgical Praxis

\section{EDV in der klinischen Chirurgie: Perspektiven}

312. HP CareVue 9000 Intensive Nursing HP CareVue 9000 System

(K. Glittenberg)

313. Expertensysteme und Entscheidungsfindung in der klinischen Praxis (C. Ohmann)

Expert Systems and Decision-making in Clinical Routine

314. Telepathology mit ISDN (Integrated Services Digital Network) (M. Oberholzer, H.-R. Fischer, H. Christen und St. Gerber)

315. Hypermedia in Aus- und Weiterbildung Hypermedia in Medical Education (F. Eitel, W. Arends und J. Kuprion)

Telepathology with Integrated Services Digital Network (ISDN) 1071

1072

\section{Neue Ansätze in der Sepsistherapie}

316. Einführung (E. Faist)

317. The Human Monoclonal Antibody HA-1A Mechanisms of Action, Spectrum of Application and Clinical Efficacy

(C. Wortel)

318. The Inactivation of TNF: From Animal Models to Clinical Reality (S. van Deventer)

319. Are there Feasible Approaches to Endothelial Protection (H. Michie)

320. Sepsis-Syndrom: Niederregulation von Monozyten und Granulozyten mit Xanthinderivaten (F. U. Schade, P. Zabel und $\mathrm{M}$. Schönharting)

321. Rational für den Einsatz von Proteasen-Inhibitoren (M. Jochum, D. Inthorn und $\mathrm{Ch}$. Waydhas)
1072

Sepsis Syndrome: Down Regulation of Monocytes and Granulocytes by Xanthine Derivatives

Rationale for the Administration of Protease-Inhibitors 
322. Hämatopoetische Wachstumsfaktoren Biologie und Wirkungsmechanismen

(K. Welte)

323. Erfahrungen mit Wachstumsfaktoren in der Neutropeniebehandlung bei Chemotherapie

(H. H. Gerhartz, M. Engelhard, G. Brittinger, G. Schlimok, D. Huhn, $\mathrm{K}$. Lennert et al.)

324. Wirkung von mikrobiellen Pathogenitätsfaktoren auf Immuneffektorzellen Einfluß von Zytokinen bei Verbrennungspatienten

(W. König, P. Müller-Lange,

M. Köller und J. Brom)

325. Einsatz von Wachstumsfaktoren bei Polytrauma

(M. Heberer, A. Marx, R. Rosso und R. Babst)

Prevention of Chemotherapy-induced

Neutropenia by Human Recombinant Granulocyte/Macrophage ColonyStimulating Factor (rhGM-CSF)

Effect of Microbial Pathogenicity Factors on Immuno-effector Cells Influence of Cytokines on Severely Burned Patients

Use of Growth Factors in the Treatment of Multiple Trauma

326. Wachstumsfaktoren im Rahmen multimodaler Therapiekonzepte solider Tumoren

The Role of Growth Factors in the Multimodality Treatment of Solid Tumors

(U. Gatzemeier)

327. Erythropoetin bei chronischen

Tumoranämien

(H. Ludwig, C. Leitgeb, E. Fritz und $\mathrm{M}$. Pecherstorfer)

Interdisziplinäre Behandlung von Weichteilgewebemalignomen Interaktives Tumorkonsil an Fallbeispielen

328. Interdisziplinäre Behandlung von Weichteilgewebemalignomen - interaktives Tumorkonsil an Fallbeispielen (K.-W. Jauch, W. Hohenberger, V. Budach, R. Issels, D. Meister, W. Mutschler, H. Steinau und $\mathrm{J}$. Treuner)

Filme und Video

Multimodality Treatment of Soft

Tissue Sarcoma - Case Presentation and Discussion
329. Operative Thorakoskopie beim Pneumothorax

(Th. Junginger, A. Heintz und Th. Böttger)

330. Die thorakoskopische thorakale Sympathektomie (W. Bauer, H. Niedermeier und U. Bergmann) 
331. Minimal invasive Lungenteilresektion Indikation, Technik und Ergebnisse (W. Wayand, R. Woisetschläger, P. Schrenk und R. Rieger)

332. Anatomie der Inguinalhernien und deren Behandlung mittels Transversalisfasziendoppelung

(W. Schweizer, M. Gilg und L. Böhlen)

333. Leistenbruchkorrektion nach Lichtenstein

(J. A. Gruwez, J. P. Puts, M. R. Christiaens, J. Deldycke und P. Nicodemus)

334. Technik der laparoskopischen Operation des Leistenbruches mit transperitoneal oder präperitoneal eingebrachtem Prolenenetz

(G. Buess, K. Manncke, J. Merhan und H. D. Becker)

335. Zerviko-mediastinale Dissektion beim differenzierten Schilddrüsenkarzinom (DTC \& MTC)

(P. E. Goretzki, D. Simon, J. Witte und H.-D. Röher)

336. Die Chirurgie des primären Hyperparathyreoidismus

(H. J. Buhr und Ch. Herfarth)

337. Adrenalektomie beim Phäochromozytom

(P. K. Wagner und M. Rothmund)

338. Die operative Behandlung des MammaKarzinoms unter Berücksichtigung brusterhaltender Verfahren

(P. Vogelbach, U. Laffer und F. Harder)

339. Technik der Leberresektion ex situ (G. Gubernatis und R. Pichlmayr)

340. Extremitätenperfusion beim malignen Melanom

(I. Krüger, R. Huber und H. J. Helling)

341. Die Bedeutung des Omentum majus für die Versorgung septischer und aseptischer Defekte (M. Pliess und Ch. Gebhardt)

342. Laparoskopische Naht- und Knüpftechniken

(A. Pier, F. Götz und M. Benedic)
Minimal Invasive Lung Resection -

Indication, Technique and Results

1081

The Anatomy of Inguinal Hernia

and its Treatment with Transversalis

Fascial Repair

1082

The Lichtenstein-Repair for Inguinal Hernia with E-PTFE

1082

Technique of Endoscopic Operation

of the Inguinal Hernia with

Transperitoneal or Preperitoneal

Prolene-mesh

1083

Cervico-mediastinal Dissection

in Differentiated Thyroid Cancer

(DTC \& MTC)

1084

Surgery of Primary

Hyperparathyroidism

1084

Adrenalectomy in Pheochromocytoma

1085

The Operative Treatment of Breast

Cancer Conservative and Amputation

1086

Technic of the Liver Resection

ex Situ

1086

Extremity Perfusion in Malignant

Melanoma

1087

1087

Suturing and Knoting Techniques

in Laparoscopy 
343. Experimenteller Einsatz neuer steuerbarer und teilautomatischer Instrumente in der endoskopischen Chirurgie (M. O. Schurr, A. Melzer, P. Dautzenberg, R. Trapp und G. Buess)

344. Laparoskopische Operationen: Fundoplikatio nach Nissen-Rossetti (G. Lepsien, F.-E. Lüdtke und T. Neufang)

345. Laparoskopische selektiv proximale Vagotomie

(B. Helms und H.-D. Czarnetzki)

346. Laparoskopische Gastro-Enterostomie (GE) zur Palliation beim inoperablen Pankreaskopfkarzinom (PKK) (R. Schlump, O. Schöb, R. Schmid und M. Röthlin)

347. Simultane laparoskopische Resektion extraluminaler Duodenaldivertikel bei Cholecystolithiasis

(D. Rühland, A. Huber und H. Schmid)

348. Laparoskopische Lymphocelenfensterung nach Nierentransplantation (V. Paolucci, W. W. Meyer, B. Schaeff und A. Encke)

349. Die laparoskopische Anlage einer Loop-Ileostomie

(T. Reck, F. Köckerling, I. Gastinger, B. Schneider, K. Bin Dayna und F. P. Gall)

350. Laparoskopische Rektopexie

(F. Köckerling, I. Gastinger,

B. Schneider, C. W. Gall, T. Reck und F. P. Gall)

351. Erweiterte Indikationen für die laparoskopische Chirurgie - Leberhämatomentfernung mit Koagulation durch Argonbeamer, Behandlung von Lymphocelen, thorakoskopische Biopsie von Mediastinal- und Lungentumoren

(K. Manncke, G. Buess, H. Raestrup und H. D. Becker)
1088

Laparoscopic Operations:

Fundoplication (Nissen-Rossetti)

1088

Laparoscopic Highly Selective

Vagotomy

1089

Laparoscopic Gastroenterostomy

(GE) for Palliation of the Inoperable Pancreatic Head Carcinoma (PHC)

1090

Simultaneous Laparoscopic Resection of Extraluminal Duodenal Diverticula in Case of Cholecystolithiasis

Laparoscopic Drainage of Lymphoceles after Renal Transplantation

1091

Laparoscopic Loop Ileostomy

Laparoscopic Rectopexy 
352. Einfluß der akuten zellulären Abstoßung auf die Leberfunktion nach orthotoper Lebertransplantation: Quantitative Funktionsuntersuchungen mit dem ${ }^{14} \mathrm{C}$-Aminopyrin-Atemtest

(J. Adolf, K. T. E. Beckurts, D. F. Müller, C. D. Heidecke, W. G. Martin, J. Schneider-Eicke und C. Wittekind)

353. Extrakorporaler Organersatz für die Leber auf der Basis organotypischer Zellkulturverfahren - Entwicklung eines Prototypen
(A. Bader und R. Pichlmayr)

354. Die postischämische Transplantatpankreatitis - Ein klinisches Modell der akuten Pankreatitis (M. Büsing, U. T. Hopt, E. Pfeffer, H. D. Becker und K. Morgenroth)

355. Manifestation von Reperfusionsschäden in der Leber mit/und ohne Organfreispülung vor der Reoxygenierung (T. Minor, W. Isselhard und J. Ziebell)

356. Laparoskopische Chirurgie: Präparation mit Hilfe der Ultraschalldissektion (K. Leber und K. Junghanns)

357. Neue Instrumente als mögliche Werkzeuge eines Intelligenten Steuerbaren Instrumentensystems ISIS (A. Melzer, M. O. Schurr, P. Dautzenberger, B. Neisius und G. Buess)

358. Neue Naht-, Knoten- und Ligaturtechniken für die endoskopische Anwendung
(A. Melzer, M. O. Schurr, B. Klemm, K. Brehl, R. Trapp und G. Buess)

359. Neues und Altbewährtes in der Chirurgie der Leistenhernien Ein Operationsverfahren in seiner historischen Entwicklung (M. Sachs und A. Encke)

360. Senkung der postoperativen Komplikationsrate nach der Implantation eines peritoneo-venösen Shunts durch ein praeoperatives Screening-Programm (A. Holzgreve, U. Sulkowski, R. H. Zimmermann und G. Hohlbach)
Effect of Acute Cellular Rejection on Liver Function Following Orthotopic Liver Transplantation: Quantitative Functional Evaluation with the ${ }^{14} \mathrm{C}$ Aminopyrine Breath Test

Organotypical Culture of Primary Hepatocytes

The Postischemic Graft Pancreatitis A Clinical Modell of Acute Pancreatitis

Reperfusion Injury in the Liver With and Without Postischemic Rinse Prior to Reoxygenation

Laparoscopic Surgery: Selective Preparation by Means of Ultrasonic Dissection

New and Established Practices in the Surgery of Inguinal Hernias - the Historical Evolution of a Surgical Procedure

Decreased Rate of Postoperative Complications After Implantation of a Peritoneo-Venous Shunt With the Help of a Preoperative Screening Score 
361. Erweiterung des konventionellen Staging durch den Mikrometastasennachweis im Knochenmark von Tumorpatienten (I. Funke, F. Lindemann, M. Heiss, K. Pantel, G. Schlimok und K.-W. Jauch)

1099

362. Zur Wertigkeit elektromanometrischer Untersuchungsverfahren in der proktologischen Funktionsdiagnostik am Beispiel der chronischen Analfissur (P. Prohm)

The Value of Electromanometry in Proctologic Function Diagnosis, for Example in Chronic Fissure in Ano

1100

Development of a Homologous Free Small Bowell Transplant for Functional Tracheal Replacement in the Animal Model

Is a Prophylaxis of Acalculous Cholecystitis with Ceruletid Possible?

Continuous veno-venous Hemofiltration $(\mathrm{CVVH})$ in Severe Necrotizing Pancreatitis (NP)

1102

The Influence of Different Prognostic Factors on the Precision of Burn Scores and the Outcome of Severely Burned Patients

Kinetics of Resorption of a New Braided PDS-Cord

368. Kunstbandfixation im Knochen durch biomorphologisches Prosthesendesign eine experimentelle Untersuchung (V. J. Wening, G. Fröschle, Ch. Tesch und $\mathrm{K}$. $\mathrm{H}$. Jungbluth)

369. Wirkung von Gelatine-Resorcin-Klebstoff auf die Aortenwand - Klinische Bedeutung (J. Ennker, H. A. Schoon, I. Ennker und R. Hetzer)

370. Prinzipien und Langzeitresultate der primären und sekundären Behandlung von Gesichtsverletzungen (J. Hoch, G. M. Lösch, M. Schrader und $\mathrm{A}$. Grewe)
Artificial ACL Osteointegration Based on Biomorphological Prothesis Design

The Impact of Gelatine-resorcinolformaldehyde-glue on the Aortic Wall - Clinical Significance

Principles and Longterm-results of Primary and Secondary Treatment of Facial Injuries 


\section{Posterausstellung}

371. Klassifikation von Keilkompressions- Classification of Wedge Compression frakturen der thorakolumbalen Wirbel- Fractures of the Thoracolumbar säule - eine neue Einteilung anhand der Spine - a Classification Based on Pathomorphologie unter Berücksichti- Pathomorphology and Stability gung der Stabilität

(A. Junge, L. Gotzen und N. Wagner)

372. Vereinfachte Behandlung von Frakturen und Luxationen der Hand durch einen neuen Faustschienenverband (H. R. Willmen, B. Mann und B. Mies)

Simplified Treatment of Bone-fractures and Joint-luxations of the Hand by Use of a new Fist-Splint-Bandage

373. Indikation, Technik und Komplikationen unterschiedlicher ,DockingManöver" beim Segmenttransport der Tibia (Ch. Josten, A. Lies, Ch. Schumann und G. Muhr)

374. Radiologische Planung und Darstellung Radiological Planning and Monitorder Charakteristika von Distraktionsosteotomien

(R. G. K. Schlenzka und M. Kasper)

375. Verbesserung der Ausbildung der Notfallversorgung Polytraumatisierter durch Nutzung paradigmatischen Trainings

(R. G. K. Schlenzka, C. Hofmann, S. Petroff-Geb und T. v. Garrel)

Indication, Technique and Complications of Different "Docking" Procedures After Bone Segment Transport

ing of Lenthening Osteotomies

Improved Instruction of Initial Management of Trauma Victims by Using Paradigmatic Training

376. Die „Critical Wound Care Unit“ Ein richtungsweisendes therapeutisches Konzept?

(H. U. Steinau, D. Hebebrand, G. Germann, W. Klein und J. Hußmann)

377. Partieller Latissimus dorsi Lappen (M. Greulich und Ch. Ludwig)

The Partial Latissimus Dorsal Flap

378. Der Glabella-Insellappen (G. B. Stark und I. M. Tangco)

The Glabella Island Flap

379. Der bikoronare Schnitt als idealer und sicherer Zugang für Rekonstruktion von Mittelgesicht und Stirn (G. B. Stark)

\section{Allgemeinchirurgie}

380. Diagnostik der Choledocholithiasis vor Cholezystektomie (M. Amlang und K. Wiedemann)

A Multivariate Analysis of the Predictive Ability of Choledocholithiasis Indicators 
381. Die distale Gallengangsligatur bei der Ratte - ein Modell für Untersuchungen bei ödematöser Pankreatitis biliärer Genese (M. Sachs, C. Gurlitt, H. Förster und A. Encke)

382. Angiodysplasia intestini P. Decker, H.-Ch. Kratzsch und A. Hirner)

383. Cumarininduzierte Darmblutung konservative Therapie (St. Trabhardt, M. Ernst, L. C. Tung und R. Häring)

384. Dekubitusprophylaxe bei Patienten mit chronischen arteriellen Durchblutungsstörungen mittels einer neu entwickelten thermoaktiven Wechseldruckmatratze

(A. Holzgreve, M. Waldner, P. W. Waldner und G. Hohlbach)

385. 10 Jahre Chirurgische Sonographie am UK Eppendorf - Wie kontrollieren wir unsere Qualität?

(C. Tesch, V. J. Wening, E. Yekebas und K.-H. Jungbluth)

386. Der Einsatz fremdblutsparender Maßnahmen in der Bundesrepublik Deutschland (alte Bundesländer) - Ergebnis einer bundesweiten Befragung (G. Singbartl und W. Schleinzer)

387. Auswirkungen der chirurgischen Fettsuchttherapie auf körperlichen und psychischen Gesundheitszustand (E. Hell und H. Roschal)

388. Komplexe Behandlungsstrategie und Qualitätssicherung in der Thoraxchirurgie - Erfahrungen mit einem neuen Informations- und Dokumentationssystem (H. Neef, H.-G. Pannwitz und $H$. Jäger)
Distal Bile Duct Ligature in Rats a Model for Investigations on Biliary Oedematous Pancreatitis

Angiodysplasia Intestini

Cumarin-induced Intestinal Bleeding - Conservative Therapy

1116

Bed Sore Prophylaxis in Patients with Chronic Arterial Occlusive Disease with a new Thermoactive Pressure Alternating Mattress

10 Years of Surgical Ultrasound at the University Hospital of Hamburg - The Way we Control the Quality of Surgical Ultrasound?

Frequency of the Application of Blood Saving Techniques in the Federal Republic of Germany

Effects of Surgery for Morbid Obesity on Physical and Mental State of Health

Complex Strategy of Treatment and Quality Control in Thoracic Surgery - Experiences with a new System of Information and Documentation

\section{Allgemeinchirurgie (Onkologie)}

389. Risiko der Rektumchirurgie im hohen Alter (K. Wellmann, T. Blankenstein und B. Ulrich) 
390. Minimal invasive Chirurgie bei Rektumtumoren - Klinische Ergebnisse (K. Kipfmüller, B. Halfar und P. Merkle)

391. Das gestielte demukosierte intestinale Transplantat zur Rekonstruktion des Beckenbodens in der Tumorchirurgie (H. Weidemann, H.-P. Lemmens, R. Lüsebrink und P. Neuhaus)

392. Die kontinenzerhaltende Proktokolektomie - Erfahrungen mit dem S-Pouch (D. Lorenz, U. Lorenz und P. Podlech)

393. Mamakarzinom des Mannes (M. Nagel, E. Hagmüller und H. D. Saeger)

394. Der Mikrometastasenstatus im Knochenmark als Entscheidungshilfe zur adjuvanten Therapie nodalnegativer Mammakarzinom-Patientinnen (I. Funke, K.-W. Jauch, S. Fries und F. W. Schildberg)

395. Behandlungsergebnisse des Ösophaguskarzinoms

(M. Nagel, H. D. Saeger und $\mathrm{M}$. Trede)

396. Ergebnisse der Magenkarzinomchirurgie 1972-1992

(D. Jentschura, H. Kopp und N. Beck)

397. Tumorbiologische Grenzen bei der Behandlung des Magenkarzinoms (J. Diermann, J. Boese-Landgraf, J. Kaiser, St. Schill, A. Scheffler, R. Häring und $H$. Ernst)

398. Leiomyosarkome des Magens Bericht über 14 Fälle (M. Peiper, A. Emmermann, S. Schröder und C. Zornig)

399. Multimodale Diagnostik- und Therapiekonzepte bei Weichteilsarkomen (B. Hoksch, H. Wolff, B. Rudolph und St. Paris)

400. Hepatectomie mit Erhaltung der Cavahinterwand - Versorgung des Retroperitoneum bei Orthotoper Lebertransplantation (R. Viebahn, W. Schareck, G. Köveker und W. Lauchart)
Demucoused Intestinal Graft for Reconstuction of the Pelvic Floor in Tumor Surgery

1122

Restorative Proctocolectomy -

Experience with the Pelvic S-Pouch

1123

Carcinoms of the Male Breast

Results of Treatment of Esophageal Cancer

Results in Surgery of Gastric Cancer 1972-1992

Tumor-biologic Limitations in the Treatment of Gastric Carcinoma

Leiomyosarcomas of the Stomach a Report About 14 Cases

Multidisziplinary Concepts in Diagnostic and Treatment of Soft Tissue Sarcoma (STS)

Preservation of Retroperitoneal Vena Cava Circumference During Recipient-Hepatectomy-Reduced Retroperitoneal Bleeding During Liver Transplantation 
401. Späte arterielle Komplikationen und ihre Therapie nach orthotoper Lebertransplantation

(J. Zanow, U. Settmacher und $\mathrm{H}$. Wolff)

\section{MIC/Endokrine und Gefäßchirurgie}

402. Die humane Plazenta: ein ideales, biologisches Modell zum Training mikrochirurgischer Gefäßanastomosen (T. Fritz, R. Simon und W. Friedl)

403. Das craniale Aneurysma nach prothetischem Ersatz des aorto-iliacalen Abschnittes

(M. Walter, H. Erasmi und

R. Schmidt)

404. Laparoskopische Lebersegmentresektion

(T. Reck, F. Köckerling, I. Gastinger und B. Schneider)

405. Ein atraumatischer Manipulator für laparoskopische Eingriffe (J. Waninger, H.-J. Mappes, R. Salm und C. Smolka)

406. Thorakoskopische Resektion eines $5 \mathrm{~cm}$ großen mediastinalen Nebenschilddrüsenadenoms

(M. Furrer, A. Leutenegger und T. Ruedi)

407. Minimale invasive endoskopische Okklusionstherapie bei Neugeborenen mit ösophagotrachealer Fistel - Experimentelle Untersuchungen zur Determinierung der intraluminalen Anwendung verschiedener Lasersysteme und Radialstrahler

(H. Meier, R. R. Lehmann und S. Czapski)

408. Senkung der Morbidität der zwei-zeitigen totalen Thyreoidektomie durch primäre Hemithyreoidektomie beim verdächtigen kalten Knoten?

(R. A. Wahl, J. Schabram, A. Luther, Ch. Luther und R. Hornstein)

409. Papilläres Mikrokarzinom der Schilddrüse: Ergebnisse nach Operation mit eingeschränkter Radikalität (A. Larena-Avellaneda, G. Doppstadt, A. Rausch und A. Larena-Avellaneda jun.)
Late Hepatic Artery Complications and Their Therapy After Liver-TX

Human Placenta: An Ideal Model for Microvascular Exercise

Suture Aneurysms After Reconstruction of the Abdominal Aorta and Iliac Vessels

Laparoscopic Liver Resection

An Atraumatic Retractor for Laparoscopic Procedures

Minimal Invasiv Endoscopic

Occlusion of ETF in Newborns -

Experimental Investigations to

Determine Intraluminell Application

in Different Laser Systems and

Radial Applicator

1133

Is Primary Total Lobectomy for Cold Thyroid Nodules Really Diminishing the Risk of Recurrent Laryngeal Nerve Paralysis with Regard to Second Stage Total Thyroidectomy?

Papillary Microcarcinoma of the Thyroid: Prognosis Following Conservative Surgical Treatment 
XLIV

Schlußveranstaltung

410. Festvortrag: „Taktstock und Skalpell“

(R. Liebermann)

Autorenverzeichnis

Sachverzeichnis 


\title{
187. Langzeitergebnisse nach Replantation von Extremitätenteilen
}

\author{
R. G. H. Baumeister, T. Hofmann, und A. Frick \\ Chirurg. Klinik u. Poliklinik, Mikro-, Hand-, wiederherst. Chirurgie, Klinikum Großhadern, \\ Universität München, Marchioninistr. 15, 81377 München
}

\section{Long-Term Follow-up after Replantation of Parts of Extremities}

\begin{abstract}
Summary. Out of 278 replanted parts of extremities, operated from January 1982 through March 1993, including 260 replantations of digits, 16 replantations of upper extremities, 2 replantations of lower extremities and an additional replantation of a penis, for the follow-up study 55 males and 7 females were investigated. They were operated between 1982 and 1987 with a minimal follow-up period of 5 years. The classification was done according to Chen. Regarding all replantations, $43 \%$ of the patients showed grade $1,36 \%$ grade $2,19 \%$ grade 3 and 1 patient showed grade $4.43 \%$ of the patients worked in their original job, $22 \%$ had to change their jobs or to be retrained, $20 \%$ were unable to work.
\end{abstract}

Key words: Replantation - Microsurgery

Zusammenfassung. Zwischen Januar 1982 und März 1993 wurden 278 Teile von Extremitäten replantiert. Darunter waren 260 Fingerreplantationen, 16 Replantationen der oberen Extremität und 2 Replantationen der unteren Extremität. Zusätzlich wurde eine Penisreplantation durchgeführt. Für die Langzeitnachuntersuchung wurden Patienten aus den Jahren 1982 bis 1987 ausgewertet; es handelte sich um 55 Männer und 7 Frauen. Es ergab sich eine Mindestnachbeobachtungszeit von 5 Jahren. Eine Klassifikation der Ergebnisse wurde in Anlehnung an Chen durchgeführt. Unter Einbeziehung aller Replantationen zeigten $43 \%$ der Patienten Grad 1, 36\% Grad 2, 19\% Grad 3. 1 Patient wies Grad 4 und damit ein funktionsloses replantiertes Extremitätenteil auf. $43 \%$ der Patienten arbeiten voll in ihrem alten Beruf, $22 \%$ befanden sich in Um- und Weiterbildung. $20 \%$ konnten keiner Arbeit mehr nachgehen.

Schlüsselwörter: Replantation - Mikrochirurgie

Im Folgenden soll über die Erfahrungen eines Replantationsdienstes mit Ergebnissen nach Replantation von Extremitätenanteilen bei einer Nachbeobachtungszeit von mehr als 5 Jahren berichtet werden. Ein Replantationsdienst führt wegen der limitierten Anzahl von Mikrochirurgen Replantationen nicht innerhalb eines Dienstplanes sondern zusätzlich zu normalen Diensten, in der Regel ohne Vergütungen durch Krankenhausträger oder Berufsgenossenschaften, aus.

Unter diesen Bedingungen wurden zwischen Januar 1982 und März 1993 insgesamt 278 Teile von Extremitäten replantiert. Darunter waren 260 Fingerreplantationen, 16 Replanta- 
Tabelle 1. Verletzungshöhe aller Finger (in der Einteilung nach Tamai)

\begin{tabular}{lllcc}
\hline \multicolumn{2}{c}{ links } & & & \multicolumn{2}{c}{ rechts } \\
\hline total & subtotal & Zone & total & subtotal \\
\hline 0 & 0 & I & 0 & 0 \\
1 & 5 & II & 0 & 7 \\
6 & 2 & III & 10 & 8 \\
6 & 9 & IV & 4 & 4 \\
0 & 7 & V & 0 & 1 \\
0 & 0 & Hand & 0 & 3 \\
4 & 5 & nicht definiert & 10 & 4 \\
17 & 29 & total & 24 & 27 \\
\hline
\end{tabular}

Zusammenfassung der Höhenlokalisation bei Fingerabtrennung in der Einteilung nach Tamai (am Daumen ist die Zone 4 nicht definiert).

Tabelle 2. Klassifikation nach Chen

\begin{tabular}{lllll}
\hline Kriterien & Grad 1 & Grad 2 & Grad 3 & Grad 4 \\
\hline Tätigkeit & alte Tätigkeit & Umschulung & $\begin{array}{l}\text { tägl. } \\
\text { Verrichtungen }\end{array}$ & \\
$\begin{array}{l}\text { Gelenk- } \\
\text { beweglichkeit }\end{array}$ & $>60 \%$ & $>40 \%$ & $>30 \%$ & $\begin{array}{l}\text { funktionsloses } \\
\text { Replantat }\end{array}$ \\
$\begin{array}{l}\text { Nerven- } \\
\text { regeneration }\end{array}$ & weitgehende & $\begin{array}{l}\text { Sensibilität im } \\
\text { Wiederherst. } \\
\text { ulned. }\end{array}$ & $\begin{array}{l}\text { teilweise } \\
\text { (Schutzsensib.) }\end{array}$ & \\
\hline
\end{tabular}

Modifizierte Gradeinteilung nach Chen zur Beurteilung der Funktion nach Replantationen.

tionen an der oberen Extremität und 2 Replantationen an der unteren Extremität. Zusätzlich wurde eine Penisreplantation durchgeführt.

Für eine Aussage zum Langzeitergebnis wurden Patienten aus den Jahren 1982 bis 1987 ausgewählt, so daß sich eine Mindestnachbeobachtungszeit von 5 Jahren ergab.

Es handelt sich um 55 Männer und 7 Frauen. Das Durchschnittsalter betrug 30,4 Jahre. Es wurden 97 Fingerreplantationen, 7 Extremitätenreplantationen sowie 1 zusätzliche Penisreplantation durchgeführt. Bei der Verletzungsursache standen die Kreissägenverletzungen an 1. Stelle. 18 Finger und 2 Extremitätenabtrennungen erfolgten auf diese Weise. Quetschtraumen stellten die zweithäufigste Abtrennungsursache dar. 8 Finger wurden auf diese Weise abgetrennt. An 3. Stelle folgten Abrißverletzungen bei Verkehrsunfällen. 2 Finger und 3 Extremitäten wurden hierbei abgerissen. Holzspaltmaschinenverletzungen, die zur Zeit in zunehmender Häufigkeit gesehen werden, waren in diesem Kollektiv für 2 Fingerabtrennungen verantwortlich.

Hinsichtlich der Höhe der Abtrennungen bei Fingerverletzungen orientierten wir uns an der Einteilung nach Tamai. Hierbei reicht Zone 1 bis zur Nagelwurzel, Zone 2 bis zum Endgelenk, Zone 3 bis zum Mittelgelenk, und Zone 4 bis zum Grundgelenk, wobei jeweils das Gelenk nicht mit einbezogen wird. Zone 5 reicht bis proximal der Metacarpaleköpfchen. Zentrale Handabtrennungen werden als solche benannt. Mischverletzungen mit unterschiedlichen Höhenlokalisationen bei einzelnen Verletzungen bezeichneten wir als nicht definiert. Es zeigte sich, daß hauptsächlich Amputationen Zone $3+4$ replantiert wurden. Praktisch nie wurden distale Verletzungen replantiert (Tabelle 1).

Die Ergebnisse wurden in Anlehnung an die Klassifikation von Chen (Tabelle 2) bewertet. Hierbei spielt die berufliche und soziale Wiedereingliederung eine dominierende Rolle. 


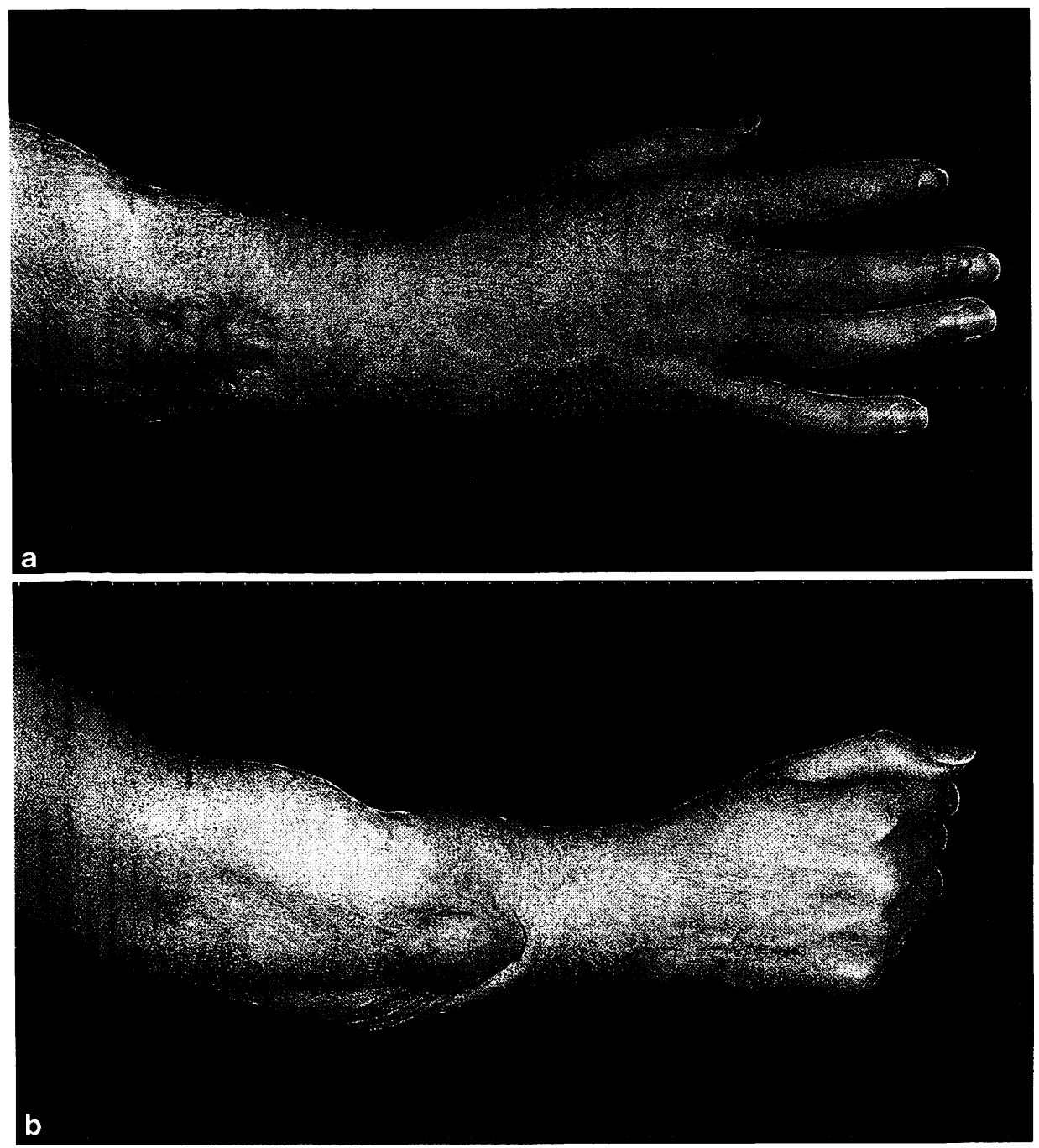

Abb. 1 a,b. Funktionsaufnahmen nach Replantation am Unterarm

Tabelle 3 a. Beurteilung der gesamten Replantationen in Anlehnung nach Chen

\begin{tabular}{lll}
\hline Chen & Anzahl & Prozent \\
\hline 1 & 18 & $43 \%$ \\
2 & 15 & $36 \%$ \\
3 & 8 & $19 \%$ \\
4 & 1 & $2 \%$ \\
\hline
\end{tabular}

Tabelle 3b. Beurteilung der Funktion nach Replantationen bei Einzelfingerverletzungen

\begin{tabular}{lll}
\hline Chen & Anzal & Prozent \\
\hline 1 & 11 & $58 \%$ \\
2 & 7 & $37 \%$ \\
3 & 1 & $5 \%$ \\
\hline
\end{tabular}

Tabelle 3c. Beurteilung der Funktion nach Replantation bei Mehrfachabtrennungen von Langfingern

\begin{tabular}{llr}
\hline Chen & Anzahl & Prozent \\
\hline 1 & 6 & $30 \%$ \\
2 & 6 & $30 \%$ \\
3 & 7 & $35 \%$ \\
4 & 1 & $5 \%$ \\
\hline
\end{tabular}



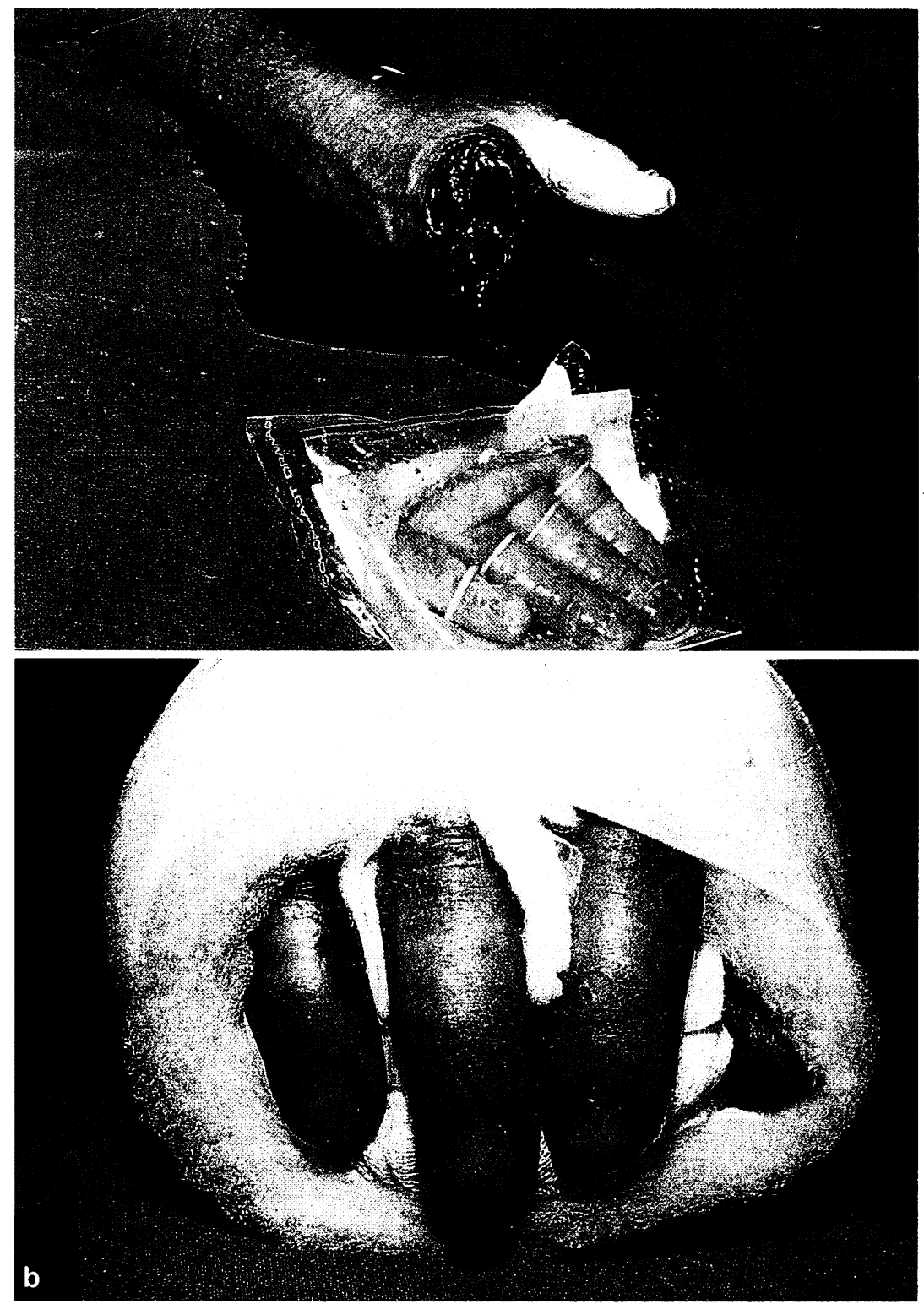

Abb. 2. a Abtrennung sämtlicher Langfinger bei einem 41jährigen Schreiner. b Frühpostoperativer Befund mit erhaltener Durchblutung

Dies ist natürlich, neben den beruflichen Anforderungen, auch abhängig vom Ausmaß der ursprünglichen Verletzung. Es wurden deshalb die Ergebnisse neben einer Gesamtbeurteilung aller Replantationen in Resultate bei Einzelfinger- und bei Mehrfach-Langfingerreplantationen unterteilt.

Es zeigte sich, daß $43 \%$ der 42 Patienten, deren Langzeitdaten zu erheben waren, mehr als 5 Jahre nach der Replantation Grad I, 36\% Grad II und 19\% Grad III erreichten. Bei einem Patienten fand sich ein funktionslos replantiertes Extremitätenteil (Tabelle $3 \mathrm{a}$ ).

Die Ergebnisse spiegeln sich auch bei der Eingliederung in die Berufswelt wider. $43 \%$ der Patienten arbeiten voll in ihrem alten Beruf, $22 \%$ befanden sich in Aus- oder Weiterbildung und $20 \%$ konnten keiner Arbeit nachgehen. 


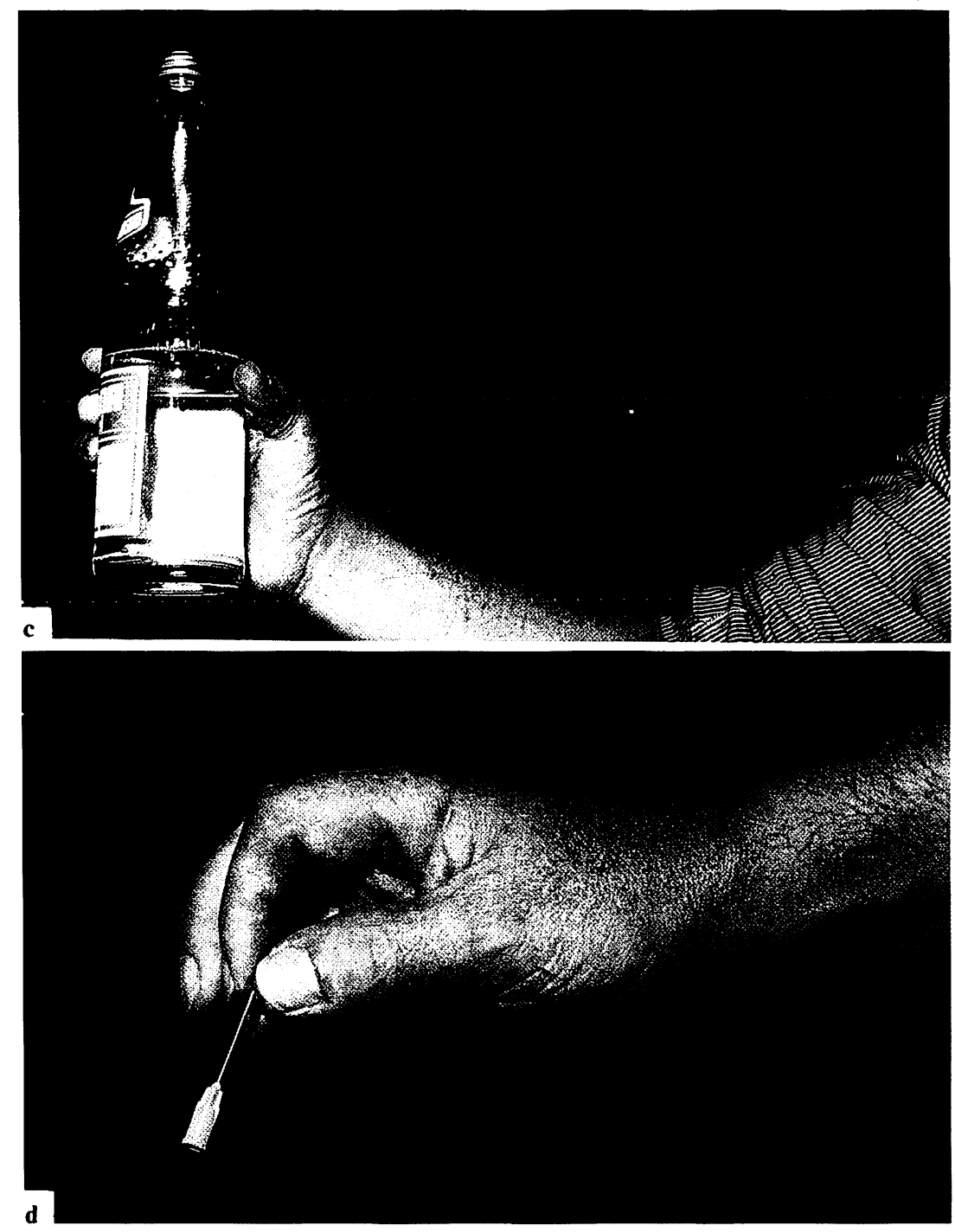

Abb. 2c,d. Späte Funktionsaufnahmen

Betrachtet man isoliert die Langzeitergebnisse der Einzel- und der Mehrfingerreplantationen (Tabelle 3b, 3c), so stellt man fest, daß doppelt so viele Patienten bei Einzelfingerreplantationen Grad I erreichen im Vergleich zu Patienten mit Mehrfingerreplantationen. Es ist dabei jedoch anzumerken, daß bei Einzelfingerreplantationen überproportional Daumenverletzungen betroffen sind. 7 Daumenreplantationen erreichten Grad I und 5 Daumenreplantationen konnten in Grad II eingruppiert werden.

Das 1. Beispiel (Abb. $1 \mathrm{a}, 1 \mathrm{~b}$ ) zeigt die Funktionstüchtigkeit einer im distalen Unterarm bei einem Verkehrsunfall abgetrennten und replantierten Hand bei einem 20jährigen Patienten. Die Hand ist wieder voll funktionstüchtig, die Sensibilität wiederhergestellt. Jetzt stören den Patienten noch die sichtbaren Narben am Unterarm.

Eine Abtrennung sämtlicher Langfinger durch eine Bandsäge bedrohte einen 41 jährigen Schreinermeister in seiner beruflichen Existenz. Das frühpostoperative Bild zeigte eine gute Durchblutung der replantierten Finger. Die Funktionsbilder zeigen sowohl die Fähigkeit 
zur feinen Manipulation als auch zur Erfassung größerer Gegenstände. Durch die Replantation konnte der Patient im weiteren Verlauf nicht nur wieder in seinem alten Betrieb arbeiten, er konnte diesen übernehmen (Abb. 2a, 2b, 2c +d).

Die Replantation eines Daumens, mehrerer Langfinger sowie von amputierten Extremitäten im Handgelenks- und distalen Unterarmbereich stellen unserer Erfahrung nach die dankbarsten Indikationen zur Replantation, auch im Hinblick auf die Langzeitergebnisse dar.

\section{Literatur}

1. Tamai S (1982) Twenty years' experience of limb replantation - Review of 293 upper extremity replants. J Hand Surg 7:549

2. Chen Z-W, Meyer VE, Kleinert HE, Beasley RW (1981) Present indications and contraindications for replantation as reflected by long-term functional results. Orthop Clin North Am 12:849 